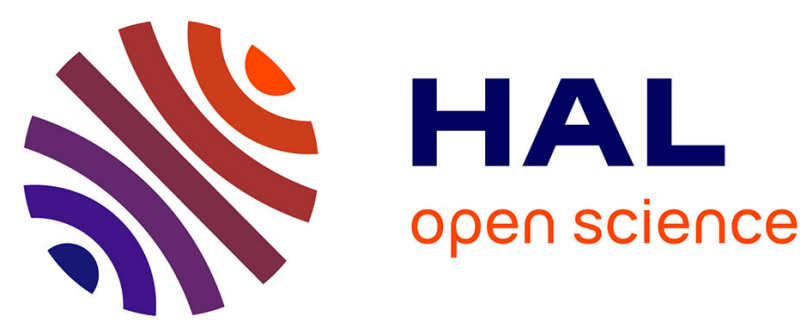

\title{
Multiscale modeling for bioresources and bioproducts
}

Marc Barnabe, Nicolas Blanc, Thomas Chabin, Jean-Yves Delenne, Agnès Duri-Bechemilh, Xavier Frank, Virginie Hugouvieux, Evelyne Lutton, Frederic Mabille, Saeid Nezamabadi, et al.

\section{- To cite this version:}

Marc Barnabe, Nicolas Blanc, Thomas Chabin, Jean-Yves Delenne, Agnès Duri-Bechemilh, et al.. Multiscale modeling for bioresources and bioproducts. Innovative Food Science \& Emerging Technologies / Innovative Food Science and Emerging Technologies , 2018, 46, pp.41-53. 10.1016/j.ifset.2017.09.015 . hal-01607608

\section{HAL Id: hal-01607608 https://hal.science/hal-01607608}

Submitted on 17 Oct 2017

HAL is a multi-disciplinary open access archive for the deposit and dissemination of scientific research documents, whether they are published or not. The documents may come from teaching and research institutions in France or abroad, or from public or private research centers.
L'archive ouverte pluridisciplinaire HAL, est destinée au dépôt et à la diffusion de documents scientifiques de niveau recherche, publiés ou non, émanant des établissements d'enseignement et de recherche français ou étrangers, des laboratoires publics ou privés.

\section{다(1)(2)}

Distributed under a Creative Commons Attribution - ShareAlikel 4.0 International 


\section{Multiscale modeling for bioresources and bioproducts}

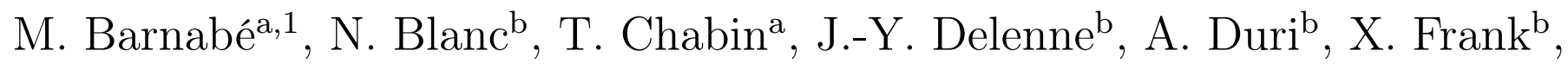

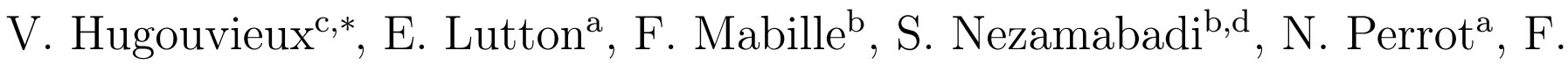
Radjai $^{\mathrm{d}, \mathrm{e}}$, T. Ruiz ${ }^{\mathrm{b}}$, A. Tonda ${ }^{\mathrm{a}}$

${ }^{a}$ GMPA, Agroparistech, INRA, Université Paris-Saclay, F-78850, Thiverval-Grignon, France

${ }^{b}$ IATE, CIRAD, INRA, Montpellier SupAgro, Université de Montpellier, F-34060, Montpellier, France

${ }^{c}$ SPO, INRA, Montpellier SupAgro, Université de Montpellier, F-34060, Montpellier, France

${ }^{d}$ LMGC, UMR 5508 - Université de Montpellier, CNRS CC048, Place Eugène Bataillon, 34095 Montpellier, France

${ }^{e}<M S E>^{2}$, UMI 3466 CNRS-MIT, CEE, MIT, 77 Massachusetts Avenue, Cambridge CA 02139, USA

\section{Abstract}

Designing and processing complex matter and materials are key objectives of bioresource and bioproduct research. Modeling approaches targeting such systems have to account for their two main sources of complexity: their intrinsic multi-scale nature; and the variability and heterogeneity inherent to all living systems. Here we provide insight into methods developed at the Food \& Bioproduct Engineering division (CEPIA) of the French National Institute of Agricultural Research (INRA). This brief survey focuses on innovative research lines that tackle complexity by mobilizing different approaches with complementary objectives. On one hand cognitive approaches aim to uncover the basic mechanisms and laws underlying the emerging collective

${ }^{*}$ Corresponding author

Email address: virginie.hugouvieux@inra.fr (V. Hugouvieux)

${ }^{1}$ Authors are listed in alphabetical order. 
properties and macroscopic behavior of soft-matter and granular systems, using numerical and experimental methods borrowed from physics and mechanics. The corresponding case studies are dedicated to the structuring and phase behavior of biopolymers, powders and granular materials, and to the evolution of these structures caused by external constraints. On the other hand machine learning approaches can deal with process optimizations and outcome predictions by extracting useful information and correlations from huge datasets built from experiments at different length scales and in heterogeneous conditions. These predictive methods are illustrated in the context of cheese ripening, grape maturity prediction and bacterial production. Keywords: Numerical modeling, Soft-matter physics, Mechanics, Microstructure, Granular matter, Hydrotextural diagram, Grain mobility, Elaboration process, Machine learning, Expert knowledge, Graphical models, Interactive learning

\section{Introduction}

Designing and processing complex matter and materials are key objectives in bioresource and bioproduct research. The complexity in this context emerges from two main sources.

One source of complexity is that when producing foods or biobased products, there are many different length scales to account for, from the molecules to macroscopic behavior and overall environmental conditions, through intermediate mesoscopic or granular particles (Fig. 1). In many cases, these length scales are coupled: for instance microstructure of raw materials strongly influences their mechanical properties, while processing conditions impact the 
rheological properties of the resulting product. These mutual dependences between length scales can be modeled in different ways and with different purposes in mind, but the key objective when modeling bioresources, foods and bioproducts is to capture the relevant length scales for the target question.

a)

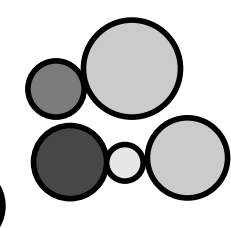

b)

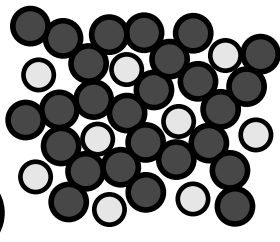

c)

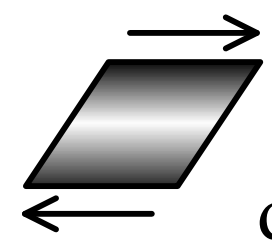

d)

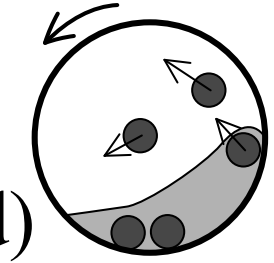

e)

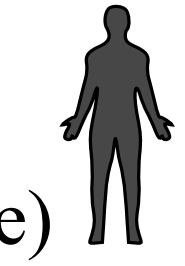

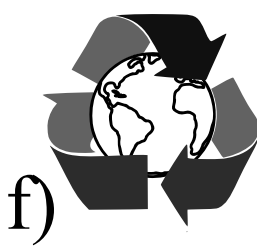

Figure 1: Characteristic length scales: a) Scale of macromolecules, colloids or grains; b) Scale of emerging microstructure; c) Scale of rheological properties (viscosity, shear strength...); d) Scale of the process with complex boundary conditions (example of a grinding mill); e) Scale of the human sensory perceptions and health considerations; f) Scale of overall environmental impacts

Another source of complexity stems from the biological origin of the raw and processed materials as bioresources (or bioproducts at a later stage of processing) show prominent variability (resulting from genetics, crop management, environmental conditions, etc) which affects their chemical composition, their architecture at different scales, and consequently their processability. Moreover, these systems are often heterogeneous, compartmented, multiphasic, or with interfaces. These variability and heterogeneity are challenging for basic disciplines such as physics, mechanics or mathematics, which are commonly used in modeling approaches. Indeed, the strongly reductionist approach they classically use is unable to capture the features responsible for the peculiar behavior of bioresources or bioproducts. Modeling approaches therefore need to bridge the gap between the well-defined monodisperse and pure systems for which well-established models exist, and the real biobased 
materials which strongly depart from this idealized representation [1, 2].

Approaches to modeling biobased systems thus need to account for both their intrinsic multiscale nature and their variability and heterogeneity. Here we focus on two classes of approaches that account for these elements of complexity using very different methods but with complementary objectives. The first type of approach aims at discovering the basic mechanisms and laws underlying the physical and mechanical behavior of biological material - whether raw or during and after processing - and it can uncover the key parameters responsible for the observed behaviors. For this purpose, different methods can be used which focus on a specific length scale and can be combined to span the whole range of length scales. On one hand, this kind of approach can be based on the description of the individual physical objects (such as macromolecules or grains) from which collective properties emerge $[3,4]$, and on the other hand it can explore the behavior of macroscopic systems from which the mechanisms responsible for the measured phenomena can emerge [5]. This approach borrows tools from physics and mechanics and can be either experimental (examples of phenomenological and heuristic approaches can be found in $[6,7]$ ) or numerical (see for instance $[8,9]$ ). By contrast, the second type of approach tackles questions dealing with the optimization of processes or the prediction of their outcome, and includes most relevant length scales in the representation of the system. This kind of approach is based on machine learning methods, which synthesize huge datasets from experiments at different scales (with factors such as genetics, physical phenomena, chemical reactions and living organisms, hence accounting for the variability of the materials) combined with expert knowledge [10]. 
Note that both the cognitive and predictive modeling approaches give insight into certain aspects of biobased systems that would otherwise be inaccessible. For instance, it is possible to deduce the physical mechanism responsible for a given phenomenon by designing a model that can represent the experimentally-obtained data. The reverse can also be done: simulation of macromolecules or grains with features known from the experiments can be used to test the experimental hypotheses and to numerically explore the collective behavior of similar systems. Moreover, the machine learning methods combining experimental and expert knowledge can effectively circumvent the fact that obtaining data in the food industry is no easy task, as experiments are expensive and many factors are involved in the processes $[11,10]$.

In the following we highlight and illustrate three approaches dealing with modeling at different length scales, and with various objectives. The intent of this paper is not to review the field of modeling in food science and biobased products but to give insight into some of the methods we currently use in this context. Section 2 introduces computational physics approaches used to gain insight into the physics of soft-matter systems and granular media, modeled as collections of generic, interacting objects from which a collective behavior emerges. We present case studies using this approach that deal with i) the self-assembly of biopolymers in solution and at interfaces (Sec. 2.4.1), ii) the structuring of biopolymers subjected to the action of enzymes (Sec. 2.4.2), iii) the rheological properties resulting from the packing of particles with different shapes (Sec. 2.4.3), and iv) the fragmentation of materials (Sec. 2.4.4). Section 3 is dedicated to the modeling of humid granular systems with the purpose of describing the multiphase and multiscale 
states subjected to (thermo)-hydro-mechanical loads applied by the process. Three case studies illustrate this experimental approach: i) dispersed multiphasic systems (Sec. 3.1 ), ii) ensiled granular media in the context of mixing processes (Sec. 3.2), and iii) humid agglomeration in couscous formation (Sec. 3.3). Finally, Section 4 deals with predictive modeling approaches applied to food processes, which are based on the use of huge experimental datasets, expert knowledge and model visualization. These methods are introduced in the case of i) Camembert ripening (Sec. 4.1), ii) grape maturity prediction (Sec. 4.2), and iii) bacterial production (Sec. 4.3). Taken together, the approaches and case studies presented here provide an overview of our research in this field.

\section{Emerging collective properties from discrete physical modeling}

How can we control the self-assembly and interfacial properties of biopolymers through the chemical nature and distribution of their building blocks? How do enzymes affect the evolution of the structuring of a biopolymer matrix? How does grinding influence the particle size distribution of a powder? How can we get an homogeneous mixture of different particles? How does composition of blends impact their rheological properties? These are some questions that have to be tackled using a discrete representation of matter as illustrated in Section 2.4.

In discrete modeling, matter is described as a set of individual objects, such as atoms, molecules, colloids undergoing Brownian motion and reciprocal interactions or grains subjected to contact and friction. At the macroscopic scale, the physical properties of such systems result from the collective behavior of the individual objects. Unlike the more classical continuous 
approaches discrete modeling gives insight into the microstructure and the relationship between the scale of the individual objects and the emerging macroscopic properties which can be measured experimentally.

\subsection{Main ingredients for discrete modeling}

In physics, modeling should be able to explain phenomena using only the main ingredients relevant to the problem, discarding the details which marginally contribute to it. This reduces the number of parameters used to describe the system, and thus allows the main causes of the observed phenomenon to be determined. For this purpose the first modeling step consists in describing the individual objects, and their interactions, whether mutual (electrostatic, capillary, friction, etc) or with their environment (thermostat, barostat, mechanical constraints, and so on).

Different types of objects are classically used, all of which rely on a more or less complex mathematical description. Figure 2a-c shows some standard examples of basic objects, which can be combined into more complex ones (such as clusters or macromolecules; see Fig. 2e,f). They can also embed internal degrees of freedom which may enable them to be deformed (Fig. 2d,f) and eventually broken (Fig. 2d).

The collective behavior of the system also depends on the interactions that act as forces and moments on the particles. These interactions can be either attractive or repulsive, short- or long-ranged. Computationally, this is described using forces (such as friction or gravity), some of which derive from potentials (e.g. electrostatic, van der Waals or covalent bonds). Figure 3 shows three examples of classical interaction pair potentials. Potentials may also rule the interactions between more than two objects, like in bond angle 
a)

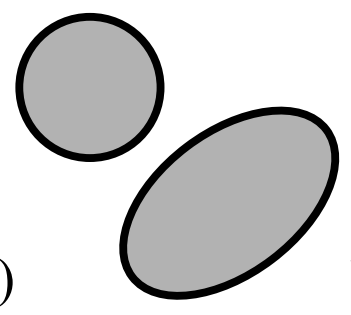

b)

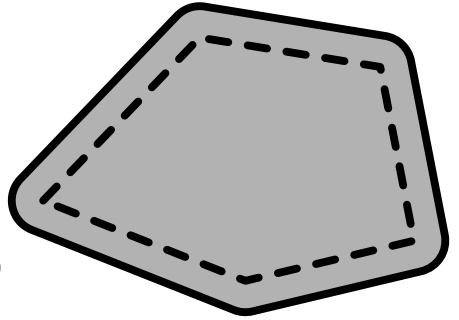

c)

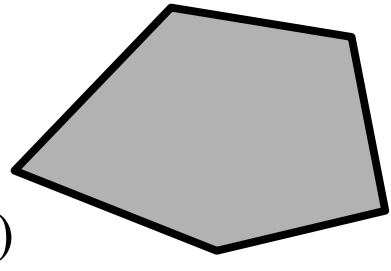

d)

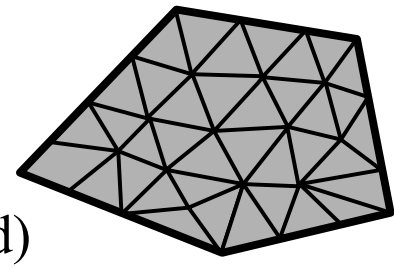

e)

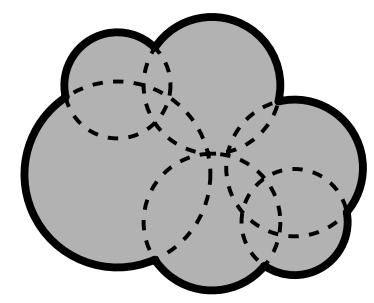

f)

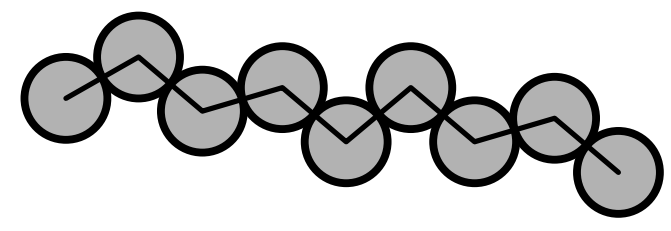

Figure 2: Schematic illustration of different types of objects used in discrete approaches. a) Sphere, ellipsoid, b) Sphero-object, c) Polygon and polyhedron, d) Meshed object, e) Cluster of simple objects, f) Chain of simple objects.

potentials (three objects) or dihedral angle potentials (four objects). Note that a tangential or rotational strength may also be considered when friction is present.

In problems where grains are in contact with interstitial phases (considered as continuous at the scale of the grain), the forces are transmitted through the interfaces and effective pair interactions are not realistic in such cases. The rheological behavior of these phases can be either liquid like for suspensions [12] or solid like for composites [13]. Discrete modeling of the grains thus has to be coupled to a specific description of interstitial phases. For example, the Lattice Boltzmann Method [12, 14] provides a versatile framework for the computation of hydrodynamic properties [15] at the subparticle scale (Fig. 4c), the simulation of capillary rise in wood [16] or droplet spreading $[17,18]$ on a powder bed (Fig. 4d).

In the simulations, interacting objects can move in a domain of space. Boundary conditions are often imposed: they can be periodic in the three 


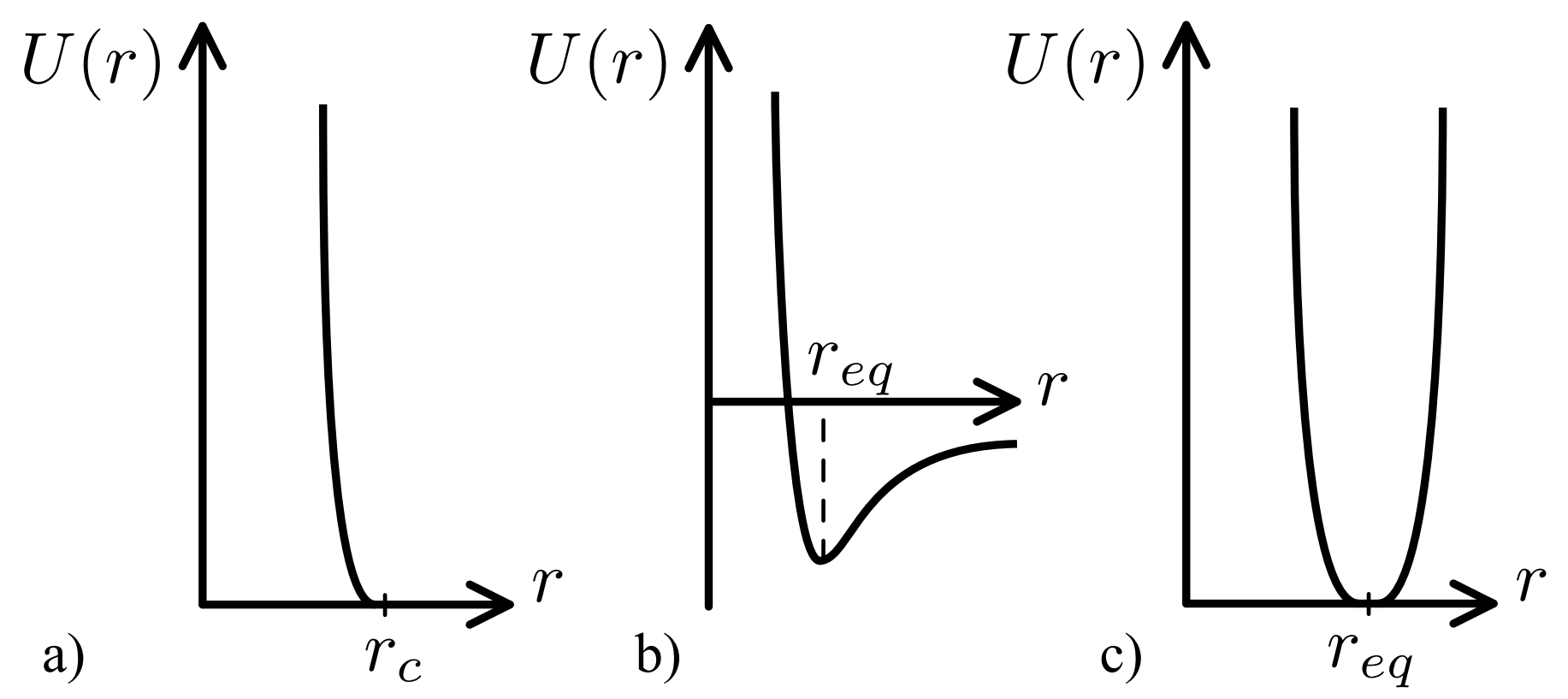

Figure 3: Examples of classical potentials. a) Potential for purely repulsive spheres; b) van der Waals potential consisting of a repulsive part (particle core) and a short-range attractive well for $r \approx r_{e q}$; c) Harmonic or higher degree potential classically used for the description of covalent bonds with an equilibrium length $r=r_{e q}$.

dimensions when the focus is on bulk properties (see the snapshots in Fig. 6), they can account for the presence of a surface or they can result from mechanical constraints (Fig. 4b,c). Figure 4a, for instance, depicts the case of a polymer solution in contact with a hydrophobic surface while in Figure 4b shearing and a normal confining pressure are applied at the top and bottom surfaces of the domain.

Whenever Brownian motion is negligible with regard to the inertia of the particles (i.e. when particle size is typically larger than $1 \mu \mathrm{m}$ ) or to the cohesive and confining forces, the evolution of the system will be strongly influenced by its initial state. For example, the flowing and compaction properties or gas permeability of a packing of particles are strongly affected by its initial density. 


\subsection{Computation}

Simulations give access to the equilibrium states of a system and/or to its evolution. For this purpose different algorithms can be used that perform iterative calculations of the positions of the objects and usually their velocities and accelerations. The Monte Carlo algorithm, for example, gives access to a set of configurations that represent the thermodynamic equilibrium of the simulated system [19]. Other methods such as molecular dynamics simulations [20], event-driven simulations or contact dynamics [21], consist in solving Newton's equations of motion and give access to the forces between the objects and to their individual trajectories. Whatever the simulation method used, computing time strongly depends on the number of interactions computed at each timestep, which is influenced by their spatial range, the complexity of the objects, and the interaction models.

It is important to note that the details in the shape of real objects and their interactions are often less relevant to the quality of the simulation than the number of simulated objects. Hence it is often necessary to simulate systems consisting of thousands or tens of thousands of objects, which means much effort has to be put into developing efficient algorithms that can run on parallel architectures (multicores, multiprocessors, GPGPU or HPC clusters) $[22,23,24]$.

Computing time also depends on temporal discretization. If the timestep is too long, the calculation is unstable, but if the timestep is too short it may unnecessarily increase computing time. The choice of timestep is guided by the physics of the problem, and should be short enough to accurately describe the fastest motions in the system, which depend on the mass of the objects 
and on the stiffness of the interactions. For example, in atomistic molecular dynamics simulations, the high energy of covalent bonds requires the use of a timestep of the order of a femtosecond $\left(10^{-15} \mathrm{~s}\right)$ which limits the total simulated time to a few nanoseconds. In contrast, when millimeter-sized objects are used as the basic units, the timestep can be of the order of a microsecond.

\subsection{Analysis of the results}

Numerical modeling of discrete objects and simulations can be seen as numerical experiments. Simulations usually produce a huge amount of data. Their processing is a central part of numerical modeling, and generally requires specific algorithms. A key advantage of the numerical approach is that it can decouple the mechanisms that are intertwined in real experiments. Indeed, discrete modeling deliberately discards part of the complexity of materials, so the mechanisms can be ranked according to their importance in the considered phenomenon. This type of modeling approach also allows the local-scale self-assembly properties to be interpreted, their time evolution to be followed, and the different regimes of structuring or destructuring of matter to be understood and mapped as a function of parameters such as temperature, polymer density, pressure or humidity $[25,26]$. In the following the discrete modeling and simulation approaches are illustrated through examples.

\subsection{Selected case studies}

\subsubsection{Self-assembly and phase-space exploration}

The self-assembly of biopolymers plays a major role in a number of applications where they are used as gelling, stabilizing or emulsifying agents. 
a)

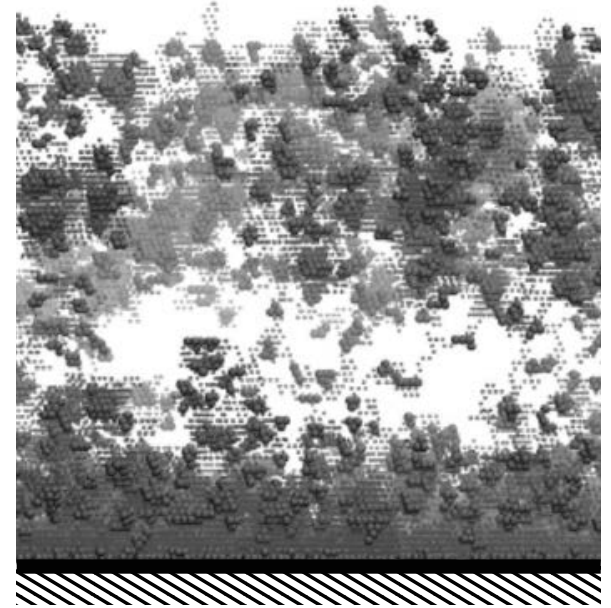

c)

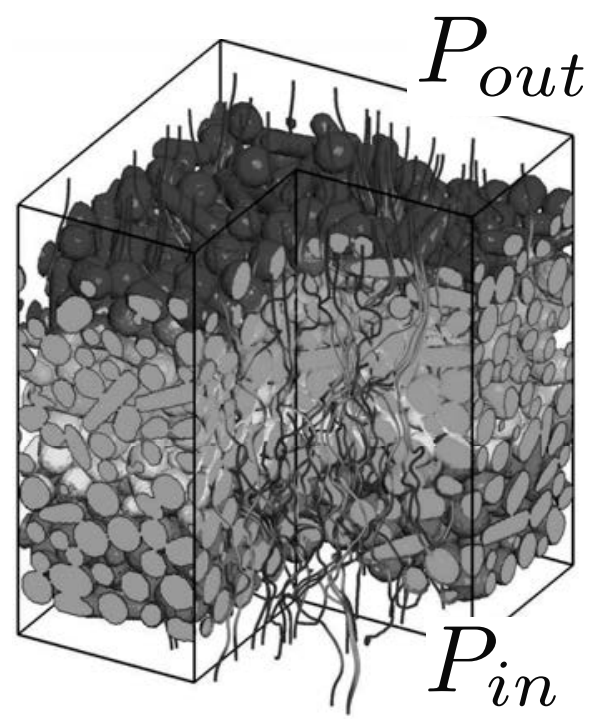

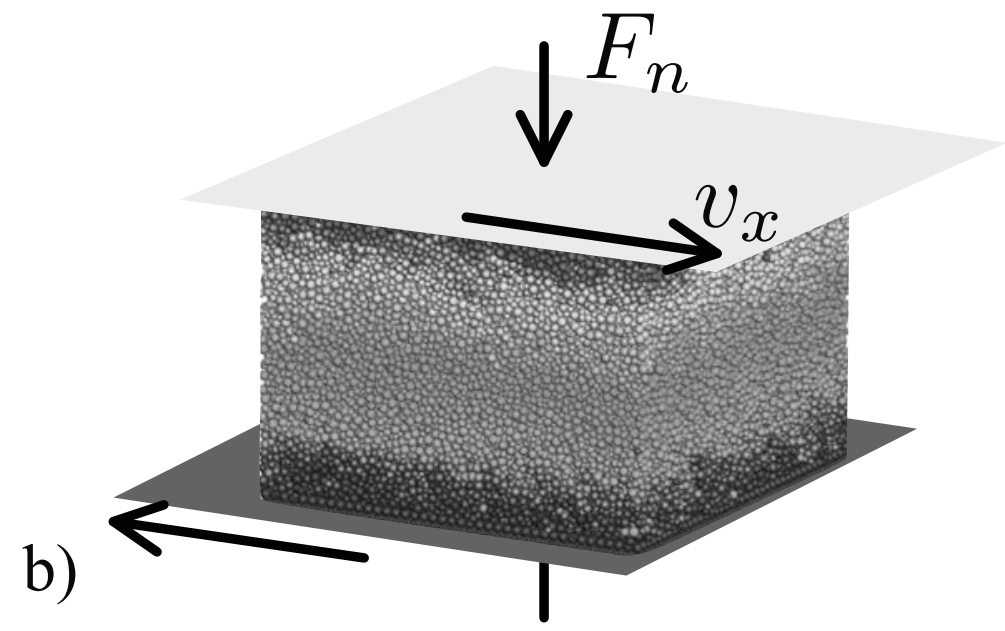

d)

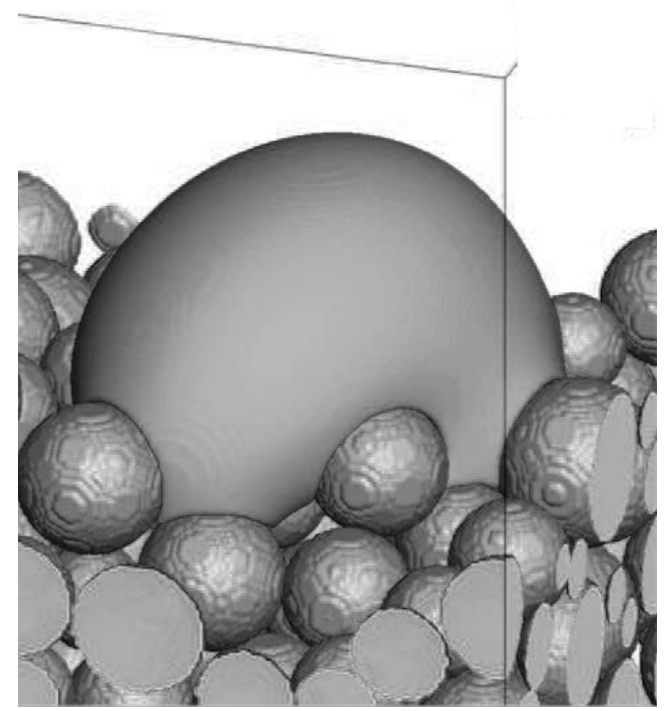

Figure 4: (color online) Some case studies. a) Solution of amphiphilic copolymers (red beads $\equiv$ hydrophobic monomers; grey beads $\equiv$ hydrophilic ones) near a hydrophobic surface; b) Shearing of a granular material between two planes. A normal force $\left(F_{n}\right)$ is applied and opposite planes slide in opposite directions at a fixed velocity $\left(v_{x}\right)$; c) Streamlines in a packing of elongated particles (fluid modeled using a lattice Boltzmann approach). A pressure gradient is applied $\left(P_{\text {in }}>P_{\text {out }}\right)$, and periodic boundary conditions are imposed in all directions; d) Spreading of a droplet on a granular layer (fluid modeled using a lattice Boltzmann approach). 
Modeling proteins or polysaccharides as amphiphilic block copolymers can provide information on how the chemical nature, distribution and interactions of monomers orchestrate their self-assembly. The simulated structures can be characterized by computing any relevant structural property available from the coordinates of the objects, such as the average distributions of core/cluster sizes (see Inset of Fig. 5), the pair distribution functions [20] or the total and partial structure factors that give access to the spatial correlations and can also be obtained from scattering experiments [27]. From these structural properties, different regimes can be determined and rationalized as a function of the parameters explored in the simulations.

For instance, using Monte Carlo simulations of multiblock copolymers (described as bead-spring chains of hydrophilic and hydrophobic monomers), we can rationalize the role of energy and entropy contributions on the equilibrium structures, in dilute and semidilute solutions or close to a surface. In dilute solutions, we evidenced the formation of a range of intramolecular structures (characterized by their structure factors) as a function of the solvent quality and the hydrophobic-to-hydrophilic balance of the copolymers [28]. In more concentrated solutions, decreasing the quality of the solvent and increasing the concentration of polymers gives rise to different structural regimes, from a homogeneous solution to a percolating network of core-shell micelles [29] (see Fig. 5), which can be in equilibrium with a 2D percolating network of micelles when the solution is in contact with a hydrophobic surface [30] (see Fig. 4a). 


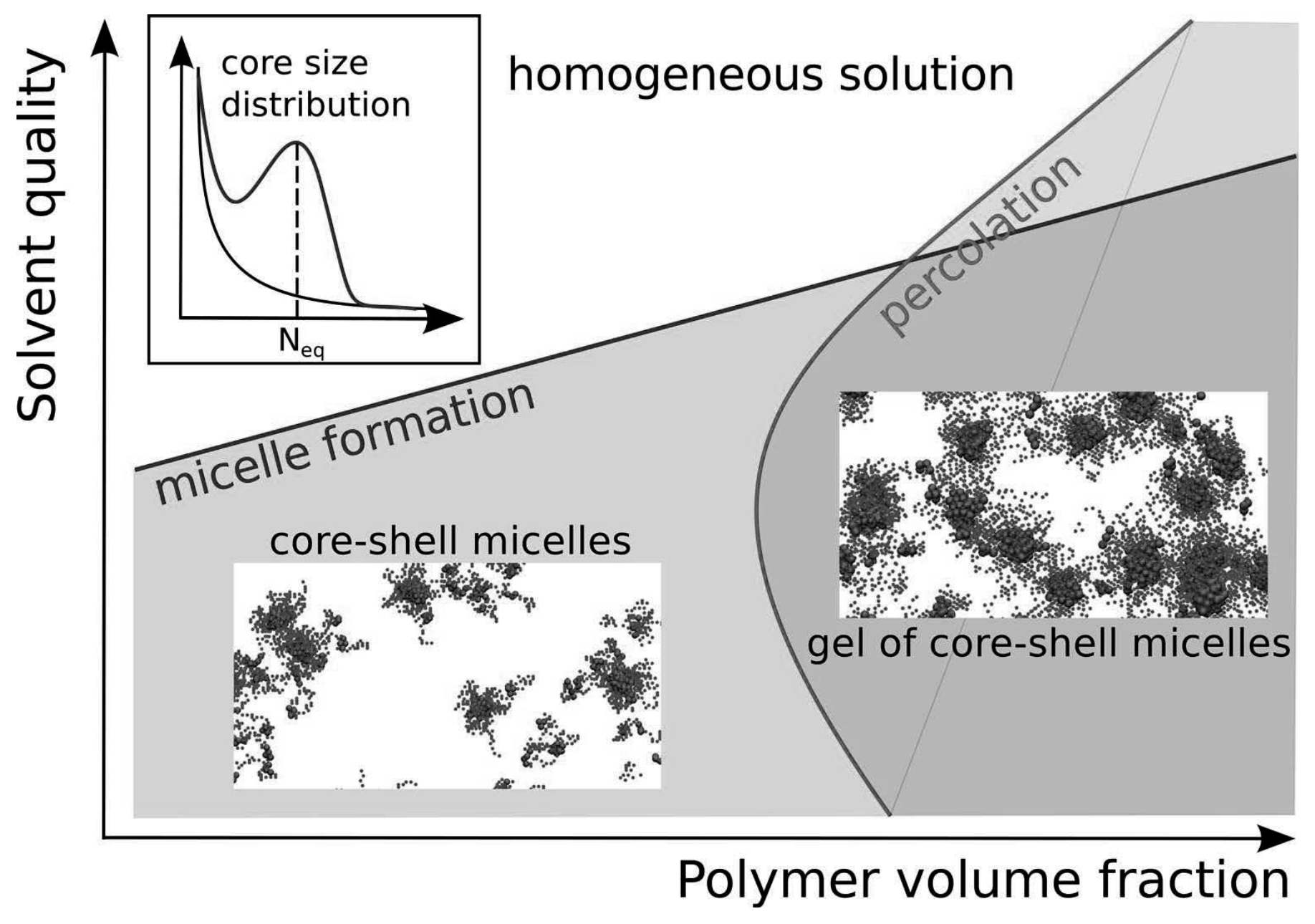

Figure 5: (color online) Influence of polymer volume fraction and solvent quality on the self-assembly of multiblock copolymers in semidilute solution (blue beads $\equiv$ hydrophilic monomers, red beads $\equiv$ hydrophobic monomers). When core-shell micelles are present (blue area), the size distribution of the hydrophobic cores shows a peak (Inset, blue curve) that is absent in homogeneous solutions (Inset, black curve). The presence of a percolating cluster of polymers (red area) is the gelation criterion. 


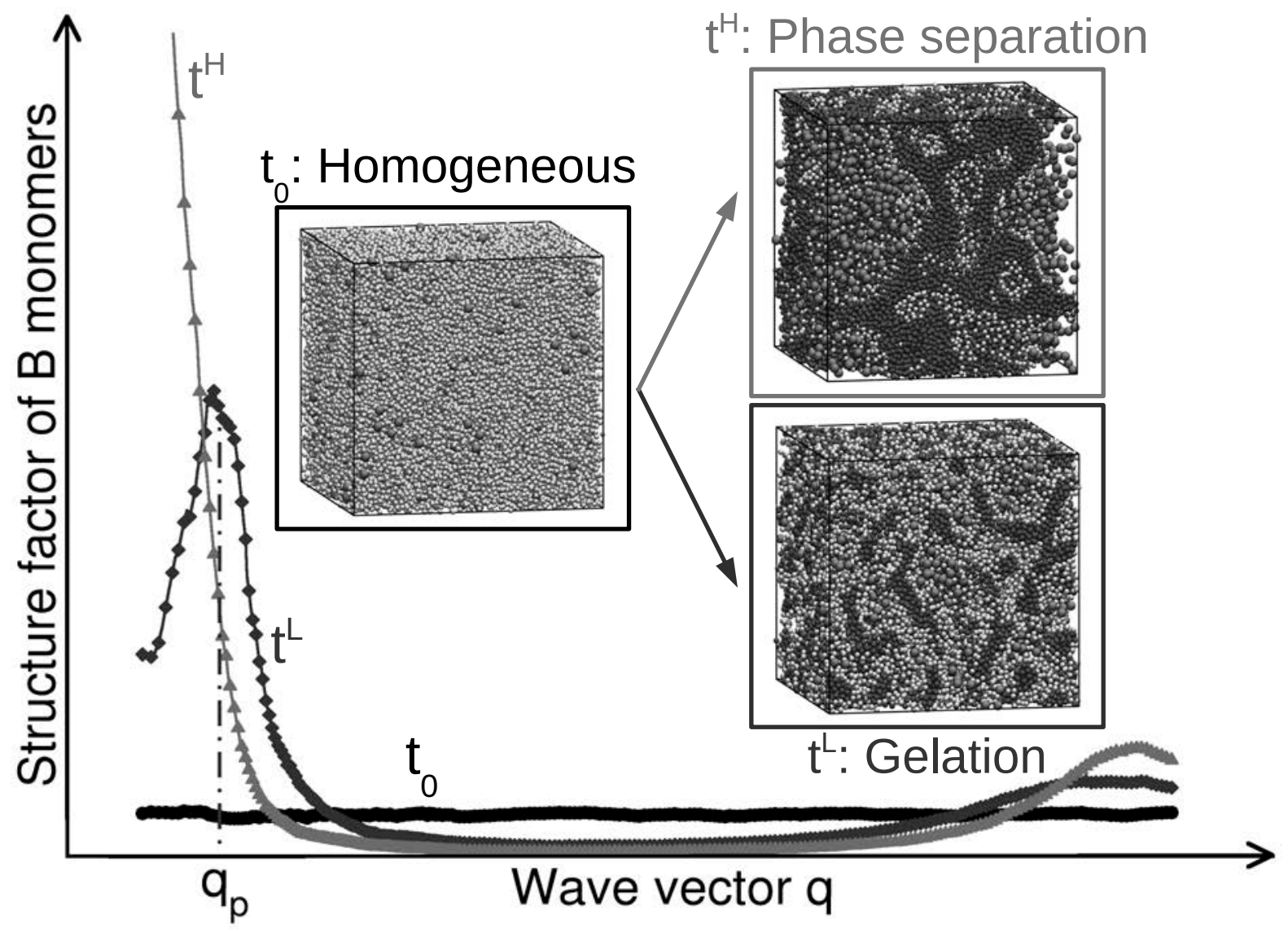

Figure 6: (color online) Simulation snapshots and partial structure factors showing the time evolution of a polymer solution under the action of enzymes (green) that convert repulsive A monomers (white) into attractive B ones (red). The system is initially homogeneous and purely repulsive $\left(t_{0}\right)$. At long times phase separation occurs at high temperature $\left(t^{H}\right.$, coarsening shown by the increasing intensity at low $\left.q\right)$ while a structured gel is formed at low temperature $\left(t^{L}\right.$, structuring shown by the correlation peak at $\left.q_{p}\right)$. 


\subsubsection{Evolution at the supramolecular scale}

During processing, the structure of materials may evolve due to constraints applied, such as temperature or pressure changes, chemical or enzymatic reactions, mechanical constraints. Time-resolved simulations give access to the evolution of such systems from an initial to a new equilibrium structure. These changes can be monitored by computing the structural properties mentioned in 2.4.1 at different times of the simulation. The time scales relevant to the observed phenomena can be extracted by comparing the structures obtained, e.g. at different temperatures or polymer concentrations. These time scales can be understood in terms of the underlying interaction potentials governing the dynamics of the system. As an example, the case of polymer solutions subjected to the action of enzymes was studied using molecular dynamics simulations [31]. In this example, enzymes, modeled as diffusing spherical objects, convert initially repulsive monomers into attractive ones, thus leading the structure of the system to evolve. For a range of polymer densities, temperatures and enzyme concentrations, the simulations allow us to follow the fraction of converted monomers over time and the evolution of the spatial correlations by computing the structure factor of the attractive B monomers (see Fig. 6).

Dynamic quantities can also be computed that give access to the diffusion properties (e.g. mean-square displacements [25]) and aging of the system, both of which are relevant in gelling and glassy materials.

\subsubsection{Packing and rheological properties}

Packing properties play an important role in processes where powders are conveyed or shaped by compaction or extrusion. In many industrial 


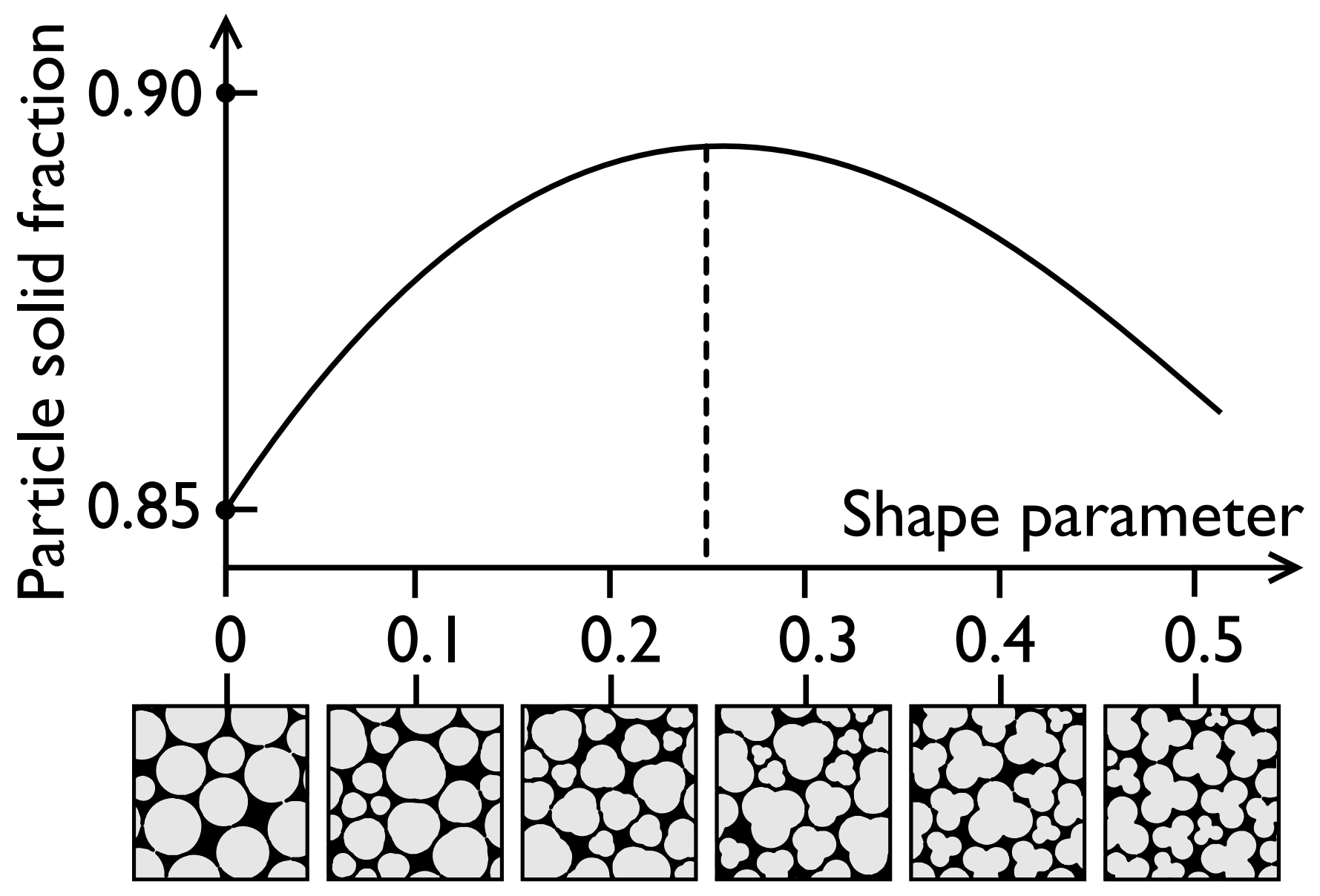

Figure 7: Computed particle solid fraction as a function of a shape parameter that increases with the deviation from the perfect sphere (for which the shape parameter equals 0 ).

applications, controlling the micro-scale fabric is a major challenge for optimizing and designing new materials: in nanoparticles, complex properties arise from grain shapes and their capacity to self-assemble; in catalysts, the specific surface and accessibility of the pore network is a major feature; in taste perception of powder-based foods, the mechanical stimuli largely depend on granulometry and apparent density. As an example, Figure 7 shows the computation by the Discrete Element Method of the evolution of the particle solid fraction of packings of dentritic particles with different shapes. This kind of abacus was used for tablets production by optimizing their density $[32,33]$.

This type of analysis was also used to investigate the rheology of powders 
as a function of their formulation. For example, the grading curve has a major influence on the processability of powders (flowability, reactivity, etc). The effect of the particle size distribution was studied in detail $[34,35]$ for purely frictional powders and for cohesive powders in which attractive interactions due to capillary, van der Waals or electrostatic forces can drastically change the energy required to structure or shape the materials under shear and confining stresses [36]. For example, understanding the hydrotextural properties of particle-liquid-gas mixtures remains a topical issue of great importance for many industrial processes involving paste or aggregate formation [37, 38]. Finally, living systems like many foods or cosmetics embed highly deformable particles. Hybrid numerical approaches have recently been developed to take advantage of both discrete and continuum approaches used for the contact and deformation, respectively, in the particle [39].

\subsubsection{Fragmentation of materials}

Comminution by cutting, crushing, grinding, and so on is one of the most important and energy-consuming unit operations in processing or chemical engineering. One of the key issue is to understand how the mechanical stresses are transmitted to particles in crushers and how the energy is dissipated to open and propagate cracks in complex raw materials embedding defects, inclusions or histological heterogeneities.

Different numerical methods have been employed to tackle this problem. The lattice-based approaches as for example the Lattice Element Method [40] or the Peridynamics Method [41], are well-suited for the computation of highly heterogeneous biomaterials. Such methods are able to simulate wave propagation through elastic brittle materials and to capture the details of 

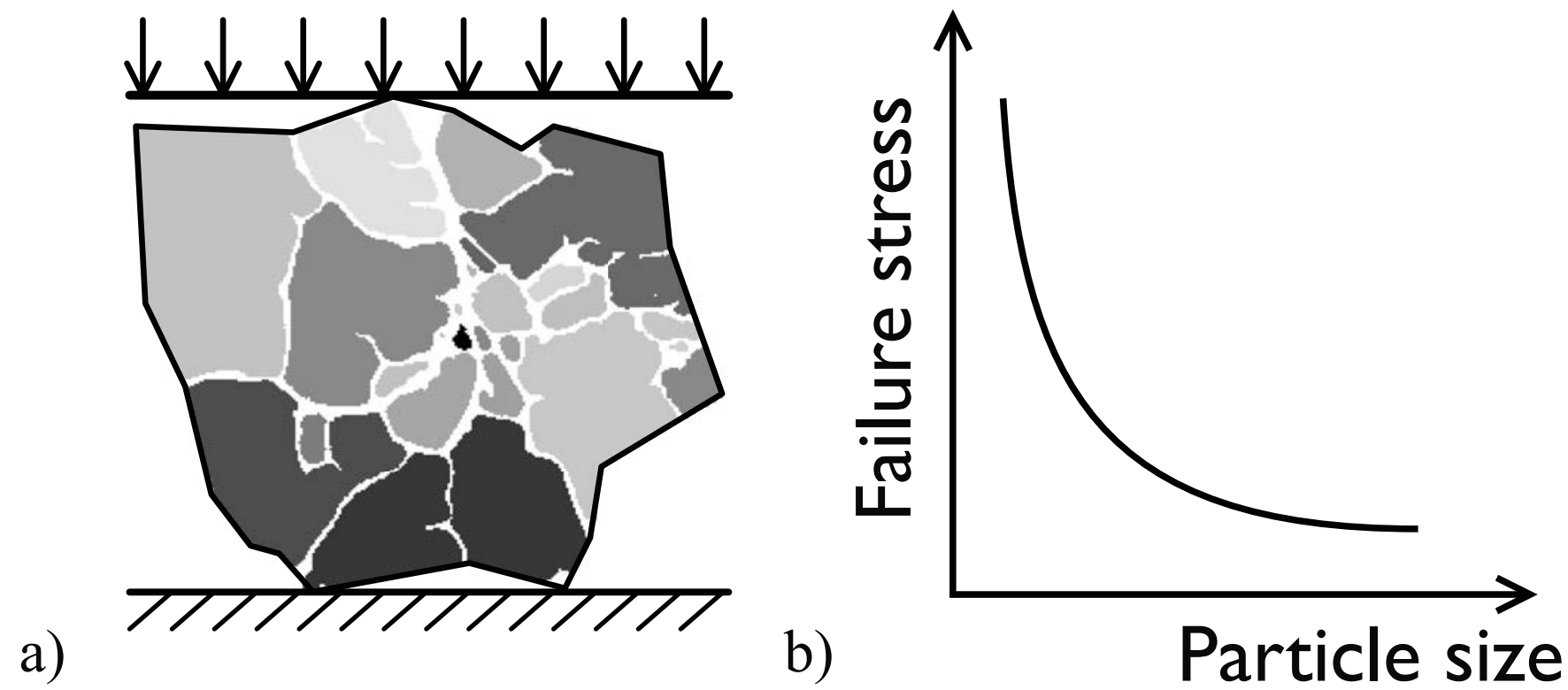

Figure 8: a) (color online) Particle fragmented using a peridynamic approach; the different fragments are colored using an image analysis algorithm. b) Scaling law between failure stress and particle size.

crack patterns. Figure 8a shows an example of a grain embedding microdefects being crushed under an impact test. The figure depicts the result of the automatic identification of the different fragments (shown in different colors) by an image analysis algorithm. Using massively parallel computing, it is possible to determine detailed statistics of the size and shapes of the fragments as a function of mechanical loading and particle microstructure. Just as in experimental results, structural defects are the preferential paths for cracks, so during a grinding process the spatial density of embedded defects in each particle decreases with their size. Consequently, the failure stress (yield force divided by the cross-section area) of a particle increases as the size of the particle decreases (Fig. 8b)). This type of scaling law is important to better understand the kinetics of comminution, especially when the objective is fine particle production. 


\section{Modeling multiphasic and multiscale granular food systems}

Food elaboration from hydrated granular media (pasta, couscous, bulgur, rice, Ebly ${ }^{\mathrm{TM}} \ldots$ ) requires the double specificity of these dispersed systems to be accounted for: (i) the solid-solid, solid-liquid, solid-liquid-gas interactions induced by the surface properties of grains (friction, capillarity...) and the thermodynamic properties of fluids, but also (ii) the 'athermal' mobility governed by the rheology of granular matter and obtained by a mechanical energy input. The food, pharmaceutical and cosmetics industries, which use many wet processing operations, involve at least one elaboration step [42], and so the control of mixing, granulation or compaction processes applied on these media remains a key challenge for optimum realization of the products. It is important to compile all the best initial component abilities and the optimal transformation processing capabilities for quality criteria linked to texture (hardness, heat transfer capacity, release of active molecules...) or to the induced rheological behavior (yield strength, flow properties) [43]. In particular, these objectives require mastering of the expression of capillary forces within the granular matrices during their hydration (agglomeration, shaping, texturization) or dehydration (drying, shrinkage) by promoting the meeting between grains and thus their mobility.

From a process point of view, i.e. at pilot scale, the technological implementation of these systems often uses dimensional analysis with operating and block diagrams [7]. Such diagrams allow the thermodynamic states of a system to be localized during its evolution under thermo-hydro-mechanical loads. They constitute an operating aid for the elaboration step by driving processes, the optimization of the formulation, and/or energy regulation. 
This approach, which is well developed in chemical and product engineering, is common when the system is essentially composed of fluid phases. The heat transfer quantification, related with mechanics and fluid rheology, facilitates an adaptation of the reactor theory and the collection of the useful functions. However, the approach is incomplete when the system is composed of dry or hydrated granular media, as their behavior deviates so much from fluid media that the associated tools are no longer suitable. It is then essential to take into account the physical properties of these discrete systems in order to bring the transformation processes under control. The case studies presented in the following are in line with this approach and contribute to providing a generic framework allowing (i) the localization of the granular system state in a 'hydrotextural' diagram and (ii) the identification of flow typologies induced by a mechanical energy input (e.g. a blade).

\subsection{Phase diagrams of dispersed multiphasic systems}

Whether it is an agglomerate, a pasta or a hard suspension, humid granular matter can be constituted of at least three dispersed phases (solid, liquid and gas) in interaction with numerous interfaces due to the discrete composition of raw matter. This complex interplay can achieve a large number of physical states. Besides their macroscopic shape, these assemblies can fundamentally be distinguished by their texture property. Indeed, according to the relative quantity of the three phases, grain arrangement depends on local capillary interactions which are the main texturation ingredients contributing to good cohesion. Capillarity, which corresponds to the depression of a liquid in the intergranular void space, plays an important role in the phenomenon of water transfer inside the granular medium, and also contributes 
to texture by mechanical rearrangement between grains. It may be strongly modulated by the surface reactivity of particles, particularly when dealing with biological material (enzymatic activity...). As an example, the sorption property of flour determines the rheological properties of pasta products, due to the structure, composition and biophysical properties of proteins and their interactions with starch, lipids and non-starch polysaccharides of flour [44].

Increasing the liquid content of the system takes it through the following sequence of states: hygroscopic pulverulent, unsaturated pulverulent, unsaturated agglomerate, saturated agglomerate, unsaturated dough, saturated pasta, hard suspension, soft suspension [45]. The unsaturated states come from the modulation of the local capillary interactions obtained by the intrinsic characteristics of the constituents and operating parameters. The different states of matter are usually obtained after a step of contact and homogenization of humidified 'dry' particles. This last step is often obtained by a wetting/mixing process followed by a texturization/shaping process, which under mechanical (compaction, extrusion...), thermic and/or hydric (cooking, drying, osmotic dehydration...) loads contributes to the formation of a granular matrix. It is therefore relevant to achieve a quantitative description of these systems that integrates multidimensional and multifactorial aspects, including grain structure, intergranular arrangement, fluid phase distribution, mixing rheology, evolution with applied loads, etc. These multiple physical states can be charted on a single phase diagram called a hydrotextural diagram (Fig. 9).

This diagram, which represents phases at a representative elementary volume scale, is comparable to diagrams that describe the "jamming" phase in 


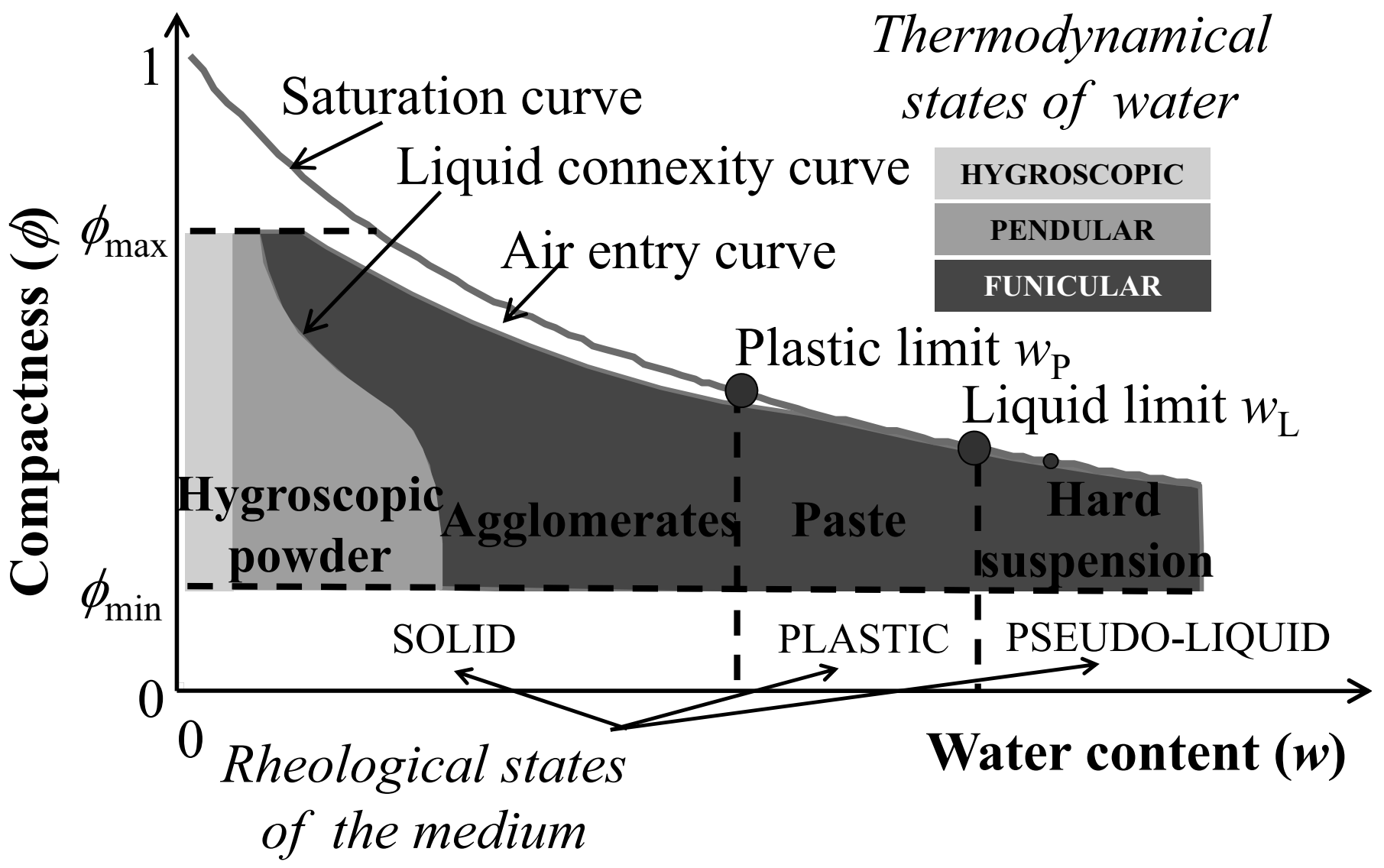

Figure 9: (color online) Hydrotextural diagram: phase diagram of humid granular media. 
soft-matter systems [46]. The hydrotextural diagram is usually represented with water content $(w)$ and compactness $(\Phi)$ axes [37, 47], and an additional axis which describes the applied thermo-hydro-mechanic load. It is limited on the upper part by the liquid saturation curve and by the maximal and minimal compactness of the granular medium (resp. close random and loose random packing). We show that the respective connected threshold of each phase is essential for understanding the transitions between the different states. In this representation, each dispersed phase may be connected or not. The connexity corresponds to the existence of a continuous path which allows to connect all the points belonging to the phase. Loose random packing represents the connexity threshold of the solid phase. The connexity of fluid phases ensures in particular their global motion under the action of a pressure gradient. The non connex case would result in the existence of drops (bubbles respectively) for the liquid phase (gas respectively) isolated from the global motion. As for the solid phase, a specific connexity threshold of each fluid phase exists, but it depends on the state of compactness. The rheological transitions are also reported on the diagram [47]: when increasing the liquid content, the 'dry' medium which is initially a solid (elastic, frictional) becomes plastic, then its behavior follows that of a hard suspension for which viscous hydrodynamic effects have the same impact as intergranular friction [45]. The phase diagram description integrates, in a coherent framework, the potential texturization linked to the presence of interstitial fluids and, in a broader sense, to the state transformation capabilities of the humid granular medium. The influence of the process parameters and fluid-solid interaction on the hydrotextural state are explicitly included in the relation- 
ship between liquid content, degree of saturation and compactness [48]. It is therefore possible to draw the transformation path of a product during its elaboration. This point is illustrated in paragraph 3.3, which presents an example dedicated to humid agglomeration.

\subsection{Mobilities promoted by a blade in an ensiled granular medium}

Most implementation processes for powders provide their motion by an external energy input. Particle flow is quite well described for mechanical tests such as in simple shear, inclined planes or rotating drum configurations $[49,50]$. Inertial and Froude numbers make it possible to represent the flow typologies (i) in the quasi-static, frictional and collisional regimes at the grain layer motion scale and (ii) in the slumping, rolling, cascading, cataracting and centrifuging regimes at reactor scale [49], respectively. These phenomenological descriptions, based on physical experimentations, have however been less investigated in the case of particle flow in semiconfined geometry and promoted by a blade or an intruder [51,52]. These flows, usually observed in food engineering during elaboration in mixers, remain relatively uncontrolled. As a rule, the humid agglomeration operations, and more globally food powder hydration are still the limiting steps of granular food elaboration industries.

The experimental characterization of these products is in our case achieved by joint measurement of (i) the force exerted on a blade by a laterally confined granular bed (Fig. 10 a) and b)) and (ii) the surface displacement field (Fig. 10 c)) obtained by Particle Image Velocimetry (PIV). Preliminary studies of the granular bed static state (i.e. without blade displacement) show that bed formation history plays an important role in force distribu- 


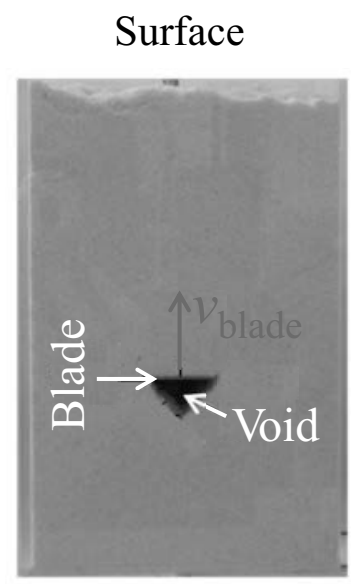

Bottom

a)

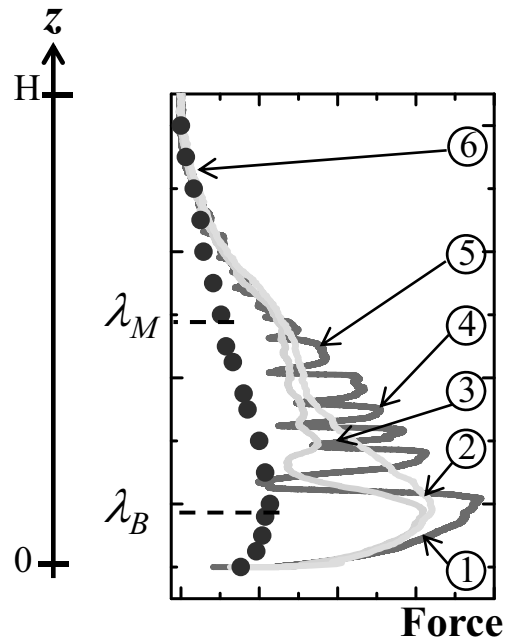

b)
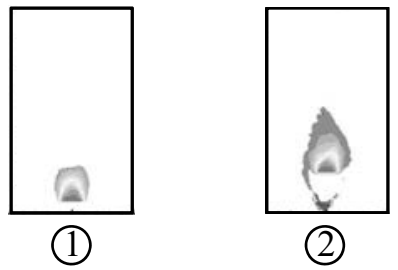

(2)

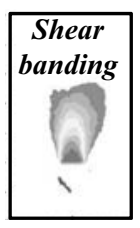

(4)

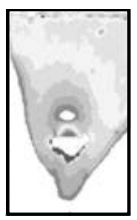

(5)

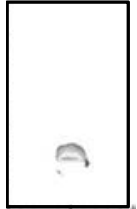

(3)

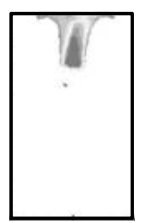

(6)

Figure 10: (color online) a) Schematic depiction of the granular bed with the blade, b) Force profiles in static (scatters) and dynamic (lines for which the fluctuations decrease with speed of the blade) conditions and c) PIV images obtained in different bed positions.

tion inside the bed [52], which can be modeled by the Janssen model [53] if the granular bed is structured by successive layers and, therefore, if the angle of repose of the granular medium is low enough [54]. Such analysis highlights the existence of two characteristic lengths: $\lambda_{M}$ which corresponds to the lateral percolation length of the intergranular force network until the cell walls, and $\lambda_{B}$ which corresponds to the force screening by the bottom of the cell (Fig. 10b)). When the system is set in motion by a blade (i.e. with blade displacement), we observe a large variety of flows that depend on type of granular medium, cell geometry and blade speed. Force profile analysis indicates the spatial location of the behavior according to the blade position in the bed and its speed. The flow, which starts from the bottom of the cell, induces a loading of the force network until it unloads (first peak in Fig. 10b)). Then, up until the percolation length $\left(\lambda_{M}\right)$, the flow is characterized by intermittent cycles of network loading and unloading, localized close to the blade in a shear banding area. The amplitude and frequency 
of these periodic fluctuations depend on blade displacement speed and type of granular media. Finally, these fluctuations decrease and vanish in the area comprised between $\lambda_{M}$ and the bed surface. Flow is then relaxed and the local stresses follow the hydrostatic state already observed in the static case [52]. Simultaneous measurement of PIV images used to calculate the vertical gradient of the velocity fields allows us to quantify grain mobility in the vicinity of the blade and in the different granular bed areas and thus to deduce the associated flows (Fig. 10c)). Close to the bottom of the cell and just above the blade, the mobility of the powder grains is reduced and homogeneous due to powder compaction and void emergence just below the blade. Under these conditions, the flow regime is almost quasi-static. In the central area, grain mobility is heterogeneous and spatially limited, and the flow regime is rather frictional [55]. In this area, grain mobility is characterized by punctual grain micro-avalanches on both sides of the blade. Close to the bed surface, the stress relaxation induces a better homogeneity of the flow, and so this last zone is adapted for mixing. It is defined by the characteristic length $\lambda_{M}$, which represents a rational tool for reactor design [53]. Spatio-temporal correlation analysis of the PIV images, which is currently performed, will more precisely qualify particle mobility, and probably also help design and drive mixing processes.

\subsection{Humid agglomeration: example of couscous formation}

The particle agglomeration process allows composite structures to be designed from smaller individual element assemblies that are brought into contact and stabilized by attractive interactions [56]. This type of elaboration process, called « bottom-up », structures the matter from the small- 
est to largest sizes. It is radically different from the « downsizing » process type where the final product is obtained by a fragmentation of larger dough pieces either by erosion and breakage [57] or by local deagglomeration [58]. In the case of the formation of couscous, a typical Mediterranean dish constituted of agglomerates of durum-wheat semolina, the goal is for the structure to emerge by the gradual increase of size. This type of elaboration requires control of the object geometry in terms of shape, size and structure. From a physico-chemical point of view, this shaping is the result of physical, mechanical and chemical processes. These phenomena are the foundation of the flow dynamics (particle motion inside mixers) and of the grain-grain and/or grain-binder-grain interaction intensity ensuring bonding between grains. For instance, semolina agglomeration into couscous grain can be assimilated to a spheroidal architecture which first implies approaching the particles closer to each other in order to link them with water droplets into small clusters: the nuclei, which interact by linking until forming an agglomerate [57]. The agglomerates are then rolled, cooked and dried to enhance their shape, mechanical strength, and long-term storability. For a defined wetting-mixing cycle (residence time, load, rotation speed, target water content, binder spraying parameters), the obtained agglomerate populations are analyzed according to their dimensional (screening and shape) and hydrotextural characteristics (compactness, water content and degree of saturation at bed-scale and agglomerates-scale). Analysis of the size distributions of agglomerates as a function of water content and the corresponding hydrotextural states shows that the structures are not saturated and the increase in size is systematically correlated with a decrease in compactness 
(Fig. 11). Whatever the mixing speed, granular media type and physicochemical properties of the binder $[59,60]$, we observe that the agglomeration process corresponds to an expansion of the agglomerates, i.e. an increase of porosity. One of the outstanding features of powder agglomeration is the nucleation/growth process [61]. We show that in the case of a low-shear mixer, the nucleation/growth process follows a fractal structuration [59], where the association mechanism is mainly due to the hydrotextural state evolution of agglomerates [62]. This morphogenesis process highlights the link between the local arrangement of phases and the emergence of the characteristic size of the product in this free shape elaboration process.

We believe that better control of particle flow inside reactors and a unified framework of hydrotextural parameters for granular media make it possible to build operating diagrams that will help innovate or improve product elaboration with a high yield and a high degree of energy efficiency.

\section{Interactive modeling for multiscale food systems}

When dealing with meaningful representations of multiscale food systems, several important issues have to be considered: data can be plagued by uncertainty, particularly when chemical, physical, and biological phenomena concur to define the process; heterogeneity of available information is also likely, as a vegetable involved in a process can be characterized by more than 40,000 genes, whereas the quality of the final product can be assessed using just a few sensory features; qualitative and quantitative information, from expert perception of food quality, to nano-properties of ingredients, may also coexist in the same process. Consequently, effective models have 


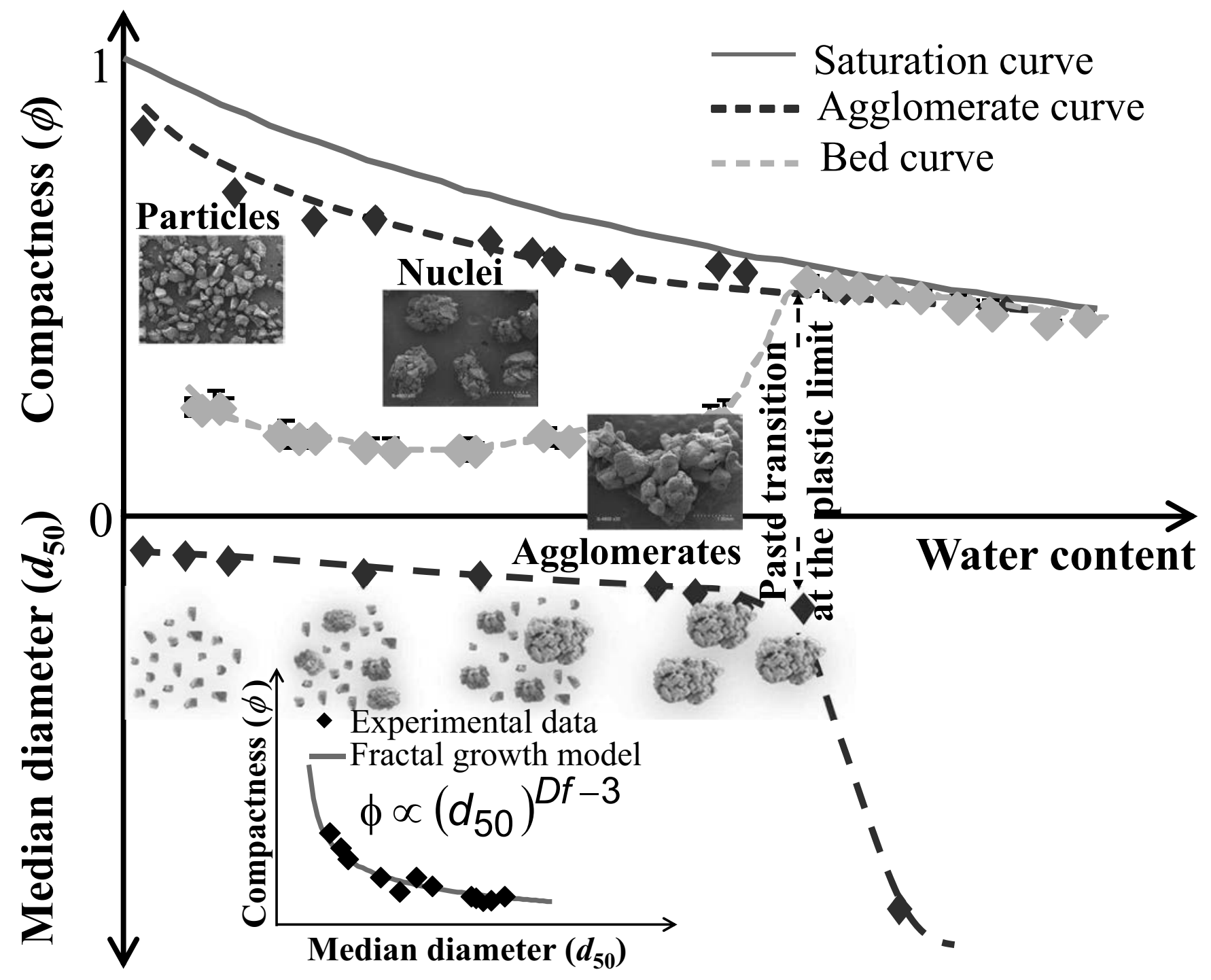

Figure 11: (color online) Hydrotextural diagram of couscous at the agglomerate scale and bed scale, and evolution of structure size as a function of water content. Inset: illustration of the fractal growth. $D f$ is the fractal dimension of agglomerates.

to account for variance, manage heterogeneous data, and be able to include both qualitative and quantitative values.

Moreover, as gathering data on bioresources and bioproducts is an expensive and time-consuming process, available datasets are often sparse and incomplete, which poses a challenge to both human modeling practitioners and machine learning algorithms. In order to obtain reliable models, it thus becomes necessary to acquire additional information from external sources. 
Experts in a specific domain can provide invaluable insight into products and processes, but this precious knowledge is often available only in the form of intuition and non-coded expertise. Including expert insight in a model is not a straightforward process, but it can effectively be tackled by having humans interacting with a machine learning process, through visualization or via specialists in encoding implicit domain knowledge [63]. In the following, three selected case studies portray different ways of combining machine learning with expert interaction.

\subsection{Dynamic Bayesian network model for Camembert ripening}

The apparent simplicity of everyday food processes often hides complex systems, where physical, chemical and living-organism processes co-exist and interact to create the final product. Cheese ripening is a major example of a process that human practitioners can achieve with success but for which several scientific details remain poorly understood. Nevertheless, even for these processes it is possible to create effective models by harnessing knowledge from experts in the domain and coupling it with experimental data by using an appropriate machine learning framework able to take into account such heterogeneous information. The work presented in [11] shows how the methodology described can be applied to the case of Camembert, a popular French cheese, creating a model that goes from micro-scale properties such as concentration of lactose and bacteria, to macro-scale properties such as color and consistency of the crust.

The approach used in this experiment is a dynamic Bayesian network (DBN) [64], a variation on a classical Bayesian network [65]. A DBN is a graph-based model of a joint multivariate probability distribution that 
captures properties of conditional independence between variables; in the graph, nodes $X_{i}(t), i=1, \ldots, N$, represent random variables, indexed by time $t$, providing a compact representation of the joint probability distribution $P$ for a finite time interval $[1, \tau]$ defined as follows:

$$
P(X(1), \ldots, X(\tau))=\prod_{i=1}^{N} \prod_{t=1}^{\tau} P\left(X_{i}(t) \mid \operatorname{Pa}\left(X_{i}\right)(t)\right)
$$

where $X(t)=X_{1}(t), \ldots, X_{N}(t)$, is called a "slice" and represents the set of all variables indexed by the same time $t . P a\left(X_{i}\right)(t)$ denotes the parents of $X i(t) . P\left(X_{i}(t) \mid P a\left(X_{i}\right)(t)\right)$ denotes the conditional probability function associated with the random variable $X_{i}(t)$ given $P a\left(X_{i}\right)(t)$. The joint probability $P(X(1), \ldots, X(\tau))$ represents the beliefs about possible trajectories of the dynamic process $X(t)$. DBNs are useful tools for combining expert knowledge with data at different levels and scales. The structure of a model can be explicitly built on the basis of expert knowledge and parameters (i.e. conditional probability functions) can be automatically learned without a priori knowledge on the basis of a dataset, all through a deterministic procedure known as parameter learning.

In this case study, two types of experts are interviewed: 4 cheesemakers with over 15 years of practice, and 8 scientists with over 10 years of research in cheese processes. The questions are carefully constructed in order to elicit expert knowledge, with methods ranging from open-ended questions to focus groups. At the same time, data is gathered from 6 experiments on the cheese ripening process, each experiment lasting 41 days, with a sampling every day. The information obtained concerns the temperature of the ripening chamber $\left(T,{ }^{\circ} \mathrm{C}\right)$, relative humidity $(R H, \%)$, and the concentration of lac- 
tose $(l o, \mathrm{~g} / \mathrm{kg})$, lactate $(l a, \mathrm{~g} / \mathrm{kg})$, and the bacteria Kluyveromyces marxianus $(K m, \mathrm{cfu} / \mathrm{kg})$, Geotrichum candidum $(G c, \mathrm{cfu} / \mathrm{kg})$, Penicillium camemberti $(P c, \mathrm{cfu} / \mathrm{kg})$, and Brevibacterium aurantiacum $(B a, \mathrm{cfu} / \mathrm{kg})$. Values of the variables are discretized in 2 to 12 classes each, depending on expert judgement [66].

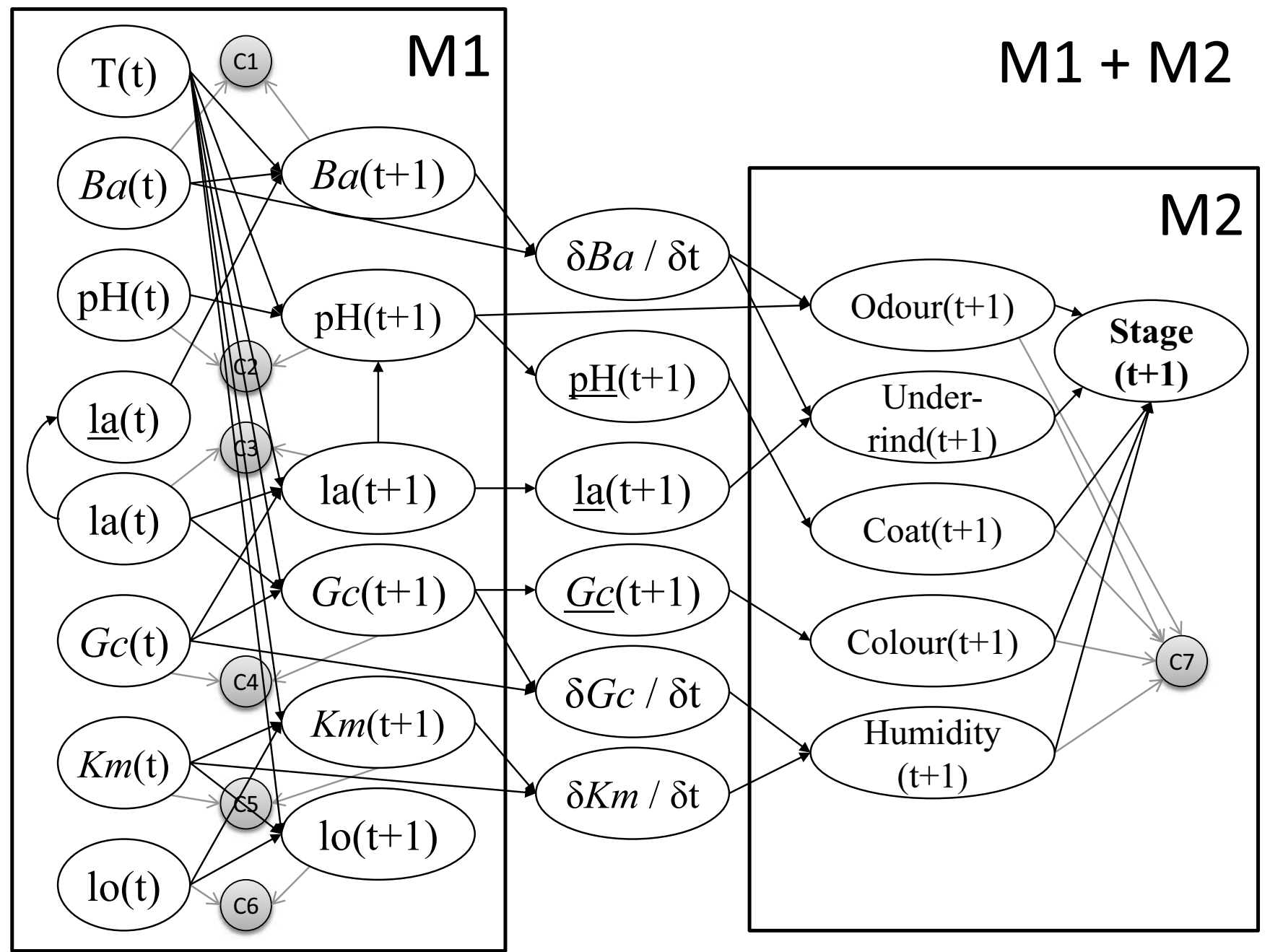

Figure 12: Final DBN model for the Camembert cheese ripening process. The part denominated M1 represents the variables taken mainly from experimental data, whereas part M2 represents variables derived from expert knowledge and assessment. Grey nodes represent constraints defined by experts.

Figure 12 shows the resulting model. Figure 13 presents an example of predictions of the dynamics in the process. From the example, it is noticeable how the model is able to satisfyingly reproduce the dynamics of variables 
tied to microbial growth, substrate consumption, and sensory properties, for different temperature conditions.
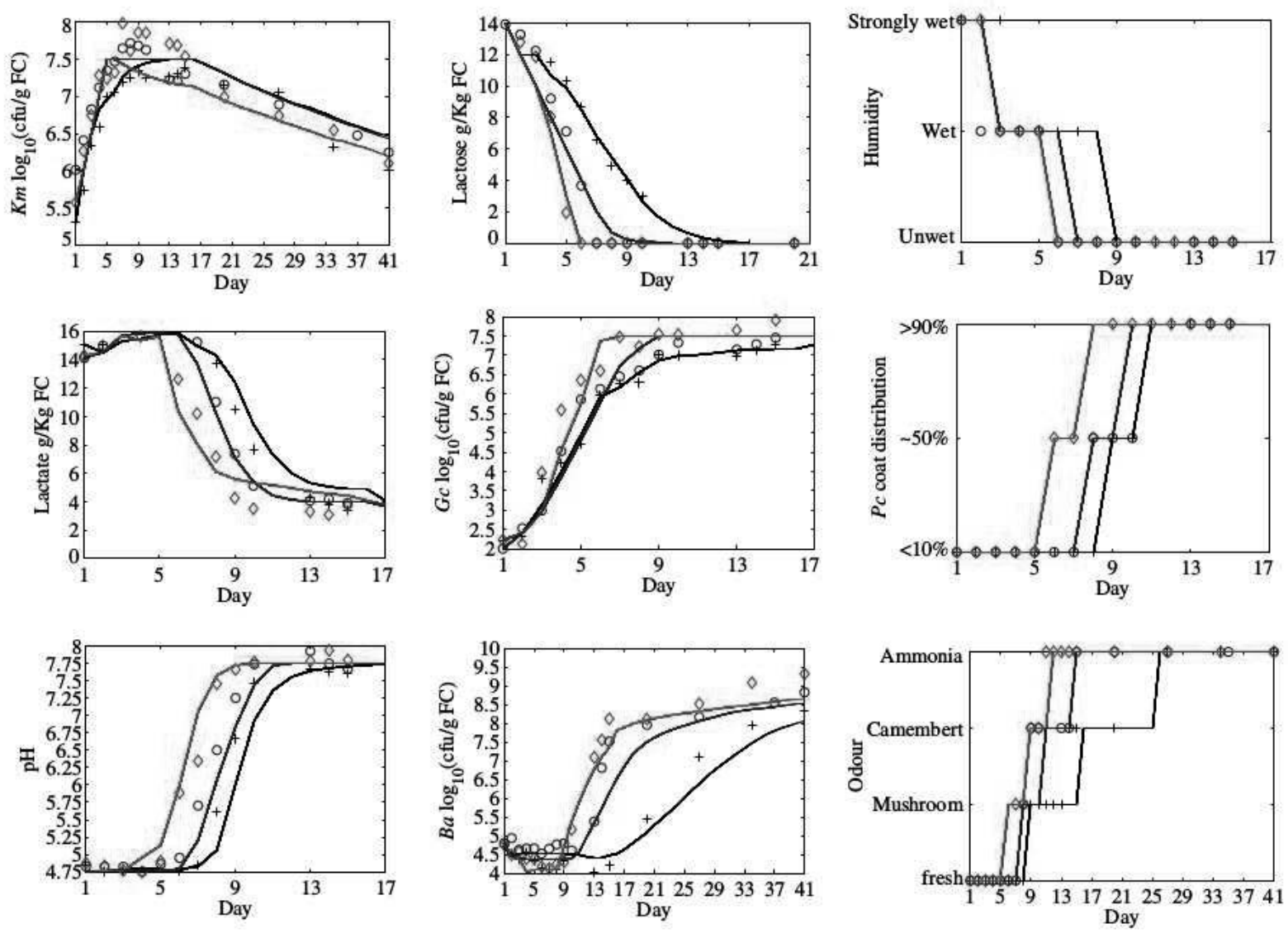

Figure 13: (color online) Predictive evolutions of (1) microbial growth ( $K m, G c, B a$ in decimal logarithm scale); (2) substrate consumption (lo, la) and (3) sensory properties ( $R H$, Pc coat and odor) obtained from DBN (line) versus raw data (points) for three different ripening processes, carried out at $8{ }^{\circ} \mathrm{C}$ (marked with + ), $12{ }^{\circ} \mathrm{C}$ (marked with o) and $16{ }^{\circ} \mathrm{C}$ (marked with $\left.\diamond\right)$.

\subsection{Decision support system for grape maturity prediction}

Predicting the right moment to harvest grapes intended for wine production is a task that traditionally is left to specialists in the field. Still, as repercussions of climate change make local weather more unpredictable, experts can use machine learning techniques as a decision support tool, helping them to deal with modified conditions. As the human knowledge gained over years of wine production is invaluable and often includes conditions that have 
not been measured in recent times, it is only sensible to include it as much as possible in the target framework. The work presented in [67] thus offers a good example of how human expertise can be employed to fill the gaps in experimental data, with the final objective of training a machine learning approach.

Data from 28 parcels of land in the Loire Valley was collected over the course of 4 years (2006-2009), giving a total of 456 data points describing weekly average temperature $\left(T,{ }^{\circ} \mathrm{C}\right)$, relative humidity $(R H, \%)$, insulation $(I n s, \mathrm{~h})$ and rainfall $(P I, \mathrm{~mm})$. Further data on sugar concentration $(S$, $\mathrm{g} / \mathrm{L})$ and acidity $\left(\mathrm{Ac}, \mathrm{g} / \mathrm{L} \mathrm{Eq} \mathrm{H}_{2} \mathrm{SO}_{4}\right)$ of the grapes is collected every two weeks, when 200 grapes randomly sampled from the parcels are crushed with a blender and subsequently analyzed. Such real-world data is expensive and time-consuming to produce, and it has to be integrated by expert knowledge, in order to improve the knowledge base eventually used for modeling. Human expertise is collected through a synthesis of the available literature and industrial reports performed by 4 scientists and 5 winegrowers working in the areas considered in the study.

As for the case study in subsection 4.1, a Dynamic Bayesian Network proves particularly suited for this application, as it makes it possible to employ qualitative and quantitative variables, at different scales, in the same model. The network is designed with the help of experts, and its structure is presented in Figure 14 (right): all the variable values are discretized into 3 classes (low, middle, high), except PIday which is divided into 4 classes (low, middle-minus, middle-plus, high). Even with an established structure, computing the parameters of each node is not trivial, as resorting to exper- 
imental data for only the four years considered would leave too many gaps. For this reason a fuzzy logic model called FGRAPE, parametrized with the previously mentioned expert knowledge, is designed and added as an input to the DBN. The complete structure of the framework, along with a prediction of the kinetics of grape maturity, is shown in Figure 14 (left). The resulting model, integrating both data and expert knowledge, is able to obtain satisfactory results, showing good $R^{2}$ values (a statistical measure of how close the data are to the fitted regression line) for both sugar content and acidity, with $R_{S}^{2}=0.82$ and $R_{A c}^{2}=0.77$, respectively.
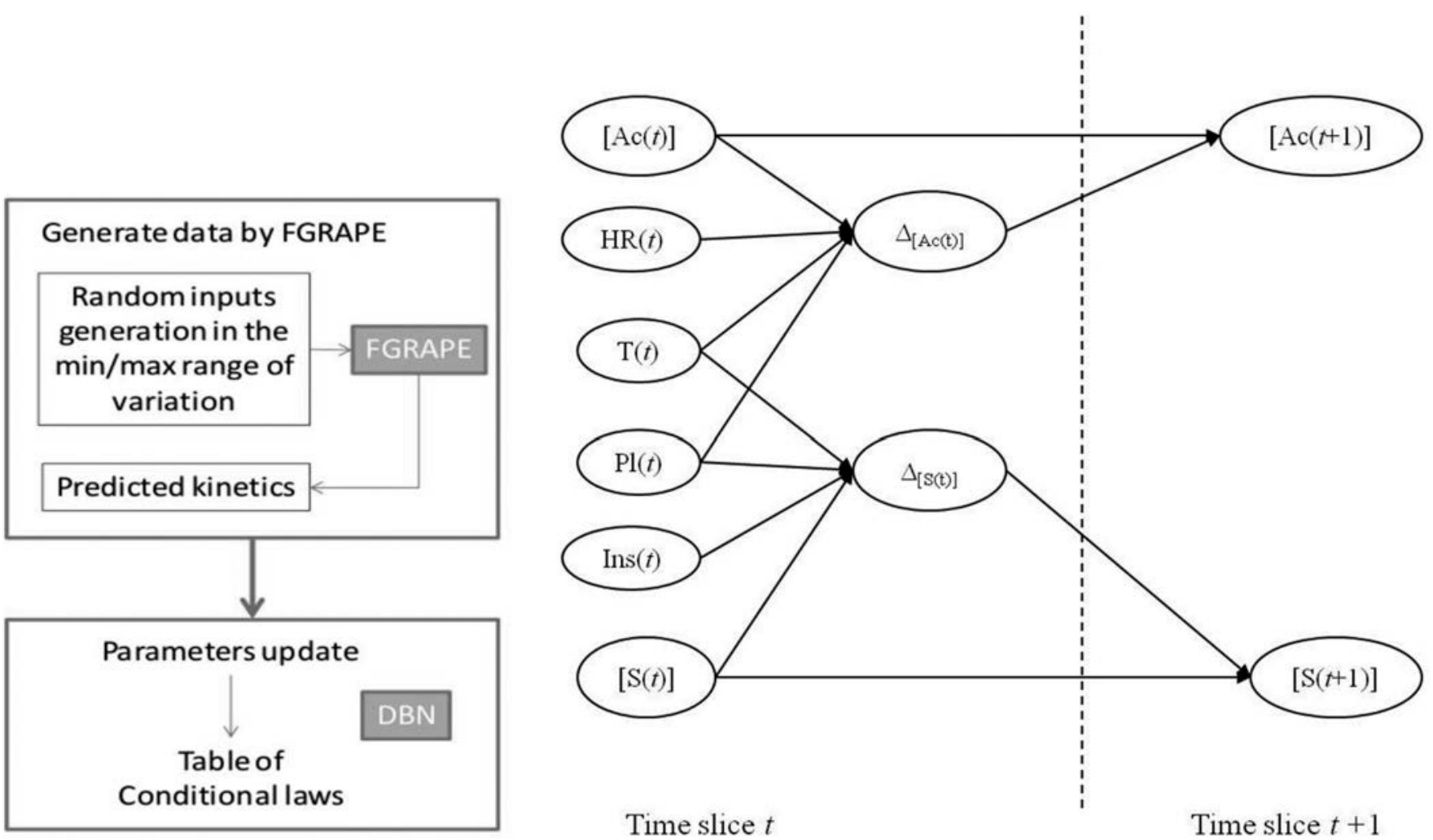

Figure 14: (color online) Proposed framework for the prediction of acidity and sugar content in grapes. (left) FGRAPE is a fuzzy logic, expert-driven tool, used to predict dynamics starting from random conditions. The data produced by FGRAPE is then used to feed the DBN, making it possible to learn robust conditional probability tables for the nodes. (right) Structure of the DBN designed for the prediction of acidity $(A c)$ and sugar content $(S)$ of grapes. 


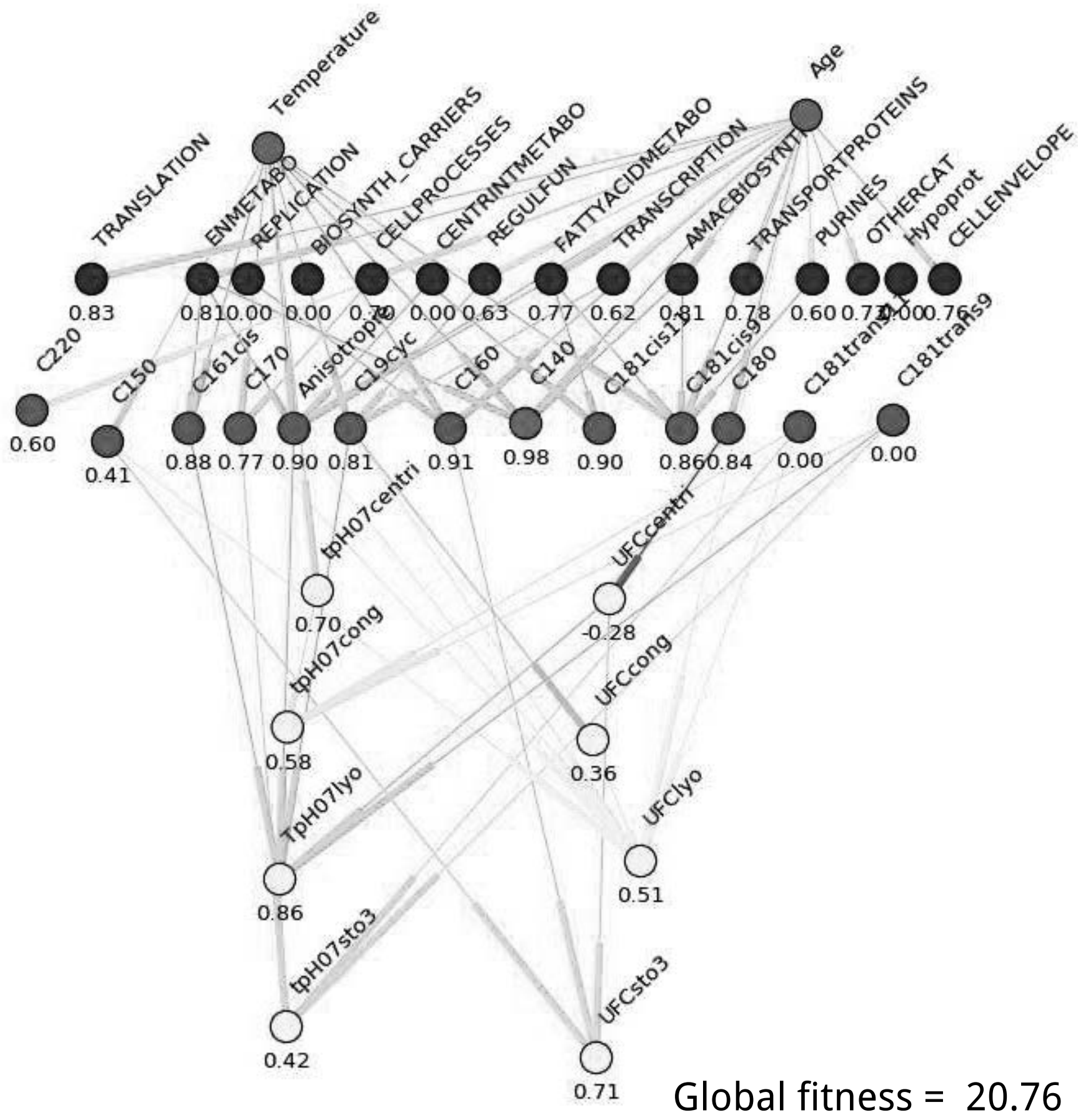

Figure 15: (color online) Graphical model generated in LIDeOGraM representing a (optimized) global model. Nodes are organized in 4 categories: experimental conditions, Genomic scale, Cellular scale, and Population scale. A Pearson correlation coefficient, calculated using the predictions from the global model compared to the experimental measurements, is printed below each node. An edge between two nodes means that the parent variable is used in the equation chosen to calculate the child variable. The color of an edge depends on the Pearson correlation coefficient, which represent the quality of the prediction. The color varies from red for a poor-quality prediction to green for a satisfying one. 
4.3. Interactive symbolic regression modeling for bacterial production and stabilization

Concentrates of Lactic Acid Bacteria (LAB) are widely used in food applications, ranging from yoghurt and cheese to fermented meat, vegetables and fruit beverages. The quality of bacterial starters, defined by their cell viability and acidification activity, depends on several control parameters, across several steps of the production and stabilization process and at different scales. Indeed, bacteria can show different levels of resistance to processes, due to the biochemical and biophysical properties and organization of their membrane [68], which in turn depend on the genomic expression of the bacteria itself. With so many possible non-linear dependencies between different scales and steps, it is not surprising that modeling the evolution of cells' quality during the production and stabilization process becomes a complex problem. Moreover, there are no models available for several subparts of this task, and even those that can be found in literature are often too simple to be included in a wider framework.

A possible solution to deal with the large number of variables and possible dependencies is to resort to automatic modeling methods, based on machine learning. The dataset in this case study concerns the full process of bacteria production and stabilisation, with 38 variables measured at 4 different steps (fermentation, concentration, freeze-drying and storage), at 4 different fermentation conditions $\left(22{ }^{\circ} \mathrm{C}\right.$ and $30{ }^{\circ} \mathrm{C}$, evaluated at the beginning of the stationary growth phase and 6 hours later). The considered variables range from transcriptomics data to fatty acid membrane composition and, from acidification activity to viability [68]. From a vast number of possible dependencies between the measured variables, an automatic methodology 
can identify the most relevant, and combine them to obtain a global model. Nonetheless, experts possess invaluable process knowledge that can considerably improve the robustness of the global model. While formalizing this often implicit knowledge is not trivial, experts' insights can be effectively included in the modeling process by resorting to interactive approaches. To achieve these objectives, we propose LIDeOGraM (Life science Interactive Development of Graph-based Models), a semi-supervised model learning framework, based on regression analysis. LIDeOGraM is able to obtain free-form equations for each variable in the process, as a function of all other variables. Each equation, describing a sub-part of the global process, can be considered a local model. Such models should fit the experimental data, and at the same time be deemed plausible by the experts. However, when using an automatic technique without expert guidelines, these two goals are often incompatible: it is always possible to find a polynomial equation that perfectly fits the data points, for example with a complex equation featuring as many parameters as data points available but such an equation could overfit the dataset, fail to represent the underlying relationship between the variables, and ultimately poorly predict the unseen data.

To avoid this issue, every variable in LIDeoGraM is associated with a set of candidate equations, obtained through symbolic regression [69]. Eureqa $^{2}$ [70], a commercial software specialized in symbolic regression, is able to obtain a set of possible equations for every variable in a given dataset. A local model can thus be associated to each variable by selecting one of the equations in the set. Symbolic regression makes it possible to effectively

\footnotetext{
${ }^{2}$ http://nutonian.com/products/eureqa/
} 
search the vast space of all possible mathematical expressions, taking into account both the fitting of the equation and its complexity - indeed, more complex equations tend to be overfitted, while simpler ones are often unable to characterize the data. A collection of local models will then constitute the base for a global model, built using an optimization algorithm, by searching for the set of local models that best fits the global dataset. To evaluate a candidate global model, the input nodes in the model are set to known experimentally-measured values, and the errors in the prediction are averaged over all nodes, thus obtaining a global error, to be minimized.

Human experts are then involved in the modeling process, via a graphical user interface, showing a node-link graph visualization of the global model, where each node represents a variable, and each link marks a possible dependency between two variables. This interface allows experts to visualize the results from Eureqa, contribute with their knowledge, and finally lead the search for an efficient global model.

For this objective, two views are available. The Local model view shows an overall qualitative view of the equation sets given by Eureqa for each variable. This view enables nodes with no satisfactory equation in terms of fitting and/or complexity to be be easily spotted. The Global model view shows the predictive capability of the current global model, for each variable. This view enables users to rapidly assess which variables in the global model are poorly predicted, but also which ones may be responsible for the poor predictions of their dependent nodes. Some results obtained for the previously described dataset [68] are presented in Figure 15.

LIDeoGraM has several ways to add expert knowledge. First, it is possible 
to attribute a category to each variable, and specify the available dependencies between categories for the symbolic regression. A category of nodes can represent a step in the process, or a scale of information. After obtaining a set of equations for every node, experts can then filter it by specifying that certain kinds of node-node dependencies are impossible. Experts can then manually add new equations in the set of candidate local models for a node, and eventually restart the search for a global model after putting all their constraints in place. With LIDeOGraM, it is possible to learn global models for the production and stabilization of bacteria. Such models can then be used to better understand how to preserve the quality of the culture during the process, foster the emergence of new hypotheses, and design new experiments, whose data could in turn be used to further improve the global model.

\section{Conclusion}

This article introduced methods and case studies intended to give insight into the abilities provided by modeling approaches in the context of bioresources and bioproducts. Choosing the appropriate method with respect to the length scale and question of interest is a crucial issue. For prediction and optimization purposes, it can be very efficient to use machine learning methods: by combining the use of experimental data and expert knowledge, they are able to account for a large number of factors affecting processes and for a number of context-relevant length scales. Contrary to these methods suited for prediction and optimization purposes, the objective of physical and mechanical modeling is to reveal the mechanisms underlying the evolution of biobased systems during processing at a given length scale. Here again, 
several methods should be combined to span the range of relevant length scales.

The case studies presented show that modeling can usefully help to control processes and the features of the resulting materials but also to propose novel paths for forming and processing materials. For instance understanding the phase transitions in granular media or revealing the dynamics of structuring of polymeric materials can serve as levers for designing original biobased materials with controlled properties, and for inventing new ways of processing bioresources.

\section{Acknowledgement}

The authors thank the PhD students, postdocs, colleagues and collaborators who contributed to some of the results cited in the Reference section. Acknowledgements to the computing platforms and to the different sources of public and industrial financial support can be found in the references to our original works.

\section{References}

[1] R. Mezzenga, P. Schurtenberger, A. Burbidge, M. Michel, Understanding foods as soft materials, Nature materials 4 (2005) 729-740.

[2] P. Shenoy, M. Viau, K. Tammel, F. Innings, J. Fitzpatrick, L. Ahrné, Effect of powder densities, particle size and shape on mixture quality of binary food powder mixtures, Powder Technology 272 (2015) 165 - 172.

[3] I. S. Aranson, L. S. Tsimring, Patterns and collective behavior in gran- 
ular media: Theoretical concepts, Reviews of modern physics 78 (2006) 641.

[4] J. Ubbink, A. Burbidge, R. Mezzenga, Food structure and functionality: a soft matter perspective, Soft Matter 4 (2008) 1569-1581.

[5] E. Rondet, M. Rundgsiyopas, T. Ruiz, M. Delalonde, J.-P. Desfours, Hydrotextural description of an unsaturated humid granular media: application to kneading, packing and drying operations, KONA Powder and Particle Journal 27 (2009) 174 - 185.

[6] G. Lebon, D. Jou, J. Casas-Vázquez, Understanding Non-equilibrium Thermodynamics: Foundations, Applications, Frontiers, SpringerVerlag, Berlin, 2008.

[7] G. Delaplace, K. Loubière, F. Ducept, R. Jeantet, Dimensional Analysis of Food Processes, ISTE Press-Elsevier, 2015.

[8] P.-M. Nguyen, W. Guiga, O. Vitrac, Molecular thermodynamics for food science and engineering, Food Research International 88, Part A (2016) $91-104$.

[9] H. Mamlouk, S. Guessasma, Finite element simulation of the compression behaviour of airy breakfast cereals, Innovative Food Science \& Emerging Technologies 19 (2013) 190 - 203.

[10] N. Perrot, H. De Vries, E. Lutton, H. G. Van Mil, M. Donner, A. Tonda, S. Martin, I. Alvarez, P. Bourgine, E. Van Der Linden, et al., Some remarks on computational approaches towards sustainable complex agrifood systems, Trends in Food Science \& Technology 48 (2016) 88-101. 
[11] M. Sicard, C. Baudrit, M. Leclerc-Perlat, P. Wuillemin, N. Perrot, Expert knowledge integration to model complex food processes. application on the camembert cheese ripening process, Expert Systems with Applications 38 (2011) 11804-11812.

[12] P. Mutabaruka, J.-Y. Delenne, K. Soga, F. Radjai, Initiation of immersed granular avalanches, Physical Review E 89 (2014) 052203.

[13] V. Topin, J.-Y. Delenne, F. Radjai, L. Brendel, F. Mabille, Strength and failure of cemented granular matter, European Physical Journal E 23 (2007) 413-429.

[14] X. Frank, P. Perré, The potential of meshless methods to address physical and mechanical phenomena involved during drying at the pore level, Drying Technology 28 (2010) 932-943.

[15] J.-Y. Delenne, V. Richefeu, X. Frank, F. Radjai, Flowbox, app deposit inra-cnrs-ujf, IDDN.FR.001.210008.000.R.A.2015.000.41000, 2015.

[16] X. Frank, G. Almeida, P. Perré, Multiphase flow in the vascular system of wood: From microscopic exploration to 3-d lattice boltzmann experiments, International Journal of Multiphase Flow 36 (2010) 599-607.

[17] X. Frank, P. Perré, Droplet spreading on a porous surface: A lattice boltzmann study, Physics of Fluids 24 (2012) 042101.

[18] X. Frank, P. Perré, H.-Z. Li, Lattice boltzmann investigation of droplet inertial spreading on various porous surfaces, Physical Review E 91 (2015) 052405. 
[19] D. Frenkel, B. Smit, Understanding molecular simulations: From algorithms to applications, Academic, San Diego, 1996.

[20] M. P. Allen, D. J. Tildesley, Computer simulation of liquids, Oxford university press, 1989.

[21] F. Radjai, F. Dubois (Eds.), Discrete-element Modeling of Granular Materials, Wiley, 2011.

[22] A. Amritkar, S. Deb, D. Tafti, Efficient parallel CFD-DEM simulations using OpenMP, Journal of Computational Physics 256 (2014) 501 - 519.

[23] M. Spellings, R. L. Marson, J. A. Anderson, S. C. Glotzer, GPU accelerated discrete element method (DEM) molecular dynamics for conservative, faceted particle simulations, Journal of Computational Physics 334 (2017) $460-467$.

[24] P. Gopalakrishnan, D. Tafti, Development of parallel DEM for the open source code MFIX, Powder Technology 235 (2013) 33 - 41.

[25] K. Binder, Monte Carlo and molecular dynamics simulations in polymer science, Oxford University Press, 1995.

[26] S. K. Kumar, J. F. Douglas, Gelation in physically associating polymer solutions, Physical Review Letters 87 (2001) 188301.

[27] J. S. Higgins, H. Benoît, Polymers and neutron scattering, Clarendon press Oxford, 1994. 
[28] V. Hugouvieux, M. A. V. Axelos, M. Kolb, Amphiphilic multiblock copolymers: From intramolecular pearl necklace to layered structures, Macromolecules 42 (2008) 392-400.

[29] V. Hugouvieux, M. A. V. Axelos, M. Kolb, Micelle formation, gelation and phase separation of amphiphilic multiblock copolymers, Soft Matter 7 (2011) 2580-2591.

[30] V. Hugouvieux, M. Kolb, Multiblock copolymer solutions in contact with a surface: Self-assembly, adsorption, and percolation, Langmuir 30 (2014) 12400-12410.

[31] V. Hugouvieux, W. Kob, Structuring polymer gels via catalytic reactions, arXiv:1608.04626 [cond-mat.soft] (2016).

[32] B. Saint-Cyr, F. Radjai, J.-Y. Delenne, P. Sornay, Cohesive granular materials composed of nonconvex particles, Physical Review E 87 (2013) 052207.

[33] E. Azéma, F. Radjai, B. Saint-Cyr, J.-Y. Delenne, P. Sornay, Rheology of three-dimensional packings of aggregates: Microstructure and effects of nonconvexity, Physical Review E 87 (2013) 052205.

[34] C. Voivret, F. Radjai, J.-Y. Delenne, M. S. E. Youssoufi, Space-filling properties of polydisperse granular media, Physical Review E 76 (2007) 021301.

[35] C. Voivret, F. Radjai, J.-Y. Delenne, M. S. El Youssoufi, Multiscale force networks in highly poly-dispserse granular media, Physical Review Letters 102 (2009) 178001. 
[36] A. Taboada, N. Estrada, F. Radjai, Additive decomposition of shear strength in cohesive granular media from grain-scale interactions, Physical Review Letters 97 (2006) 098302.

[37] T. Ruiz, M. Delalonde, B. Bataille, G. Baylac, C. Dupuy, Texturing unsaturated granular media submitted to compaction and kneading processes, Powder Technology 154 (2005) 43-53.

[38] J.-Y. Delenne, V. Richefeu, F. Radjai, Liquid clustering and capillary pressure in granular media, Journal of Fluid Mechanics 762 (2015) R5.

[39] S. Nezamabadi, F. Radjai, J. Averseng, J.-Y. Delenne, Implicit frictional-contact model for soft particle systems, Journal of the Mechanics and Physics of Solids 83 (2015) 72-87.

[40] R. Affes, V. Topin, J.-Y. Delenne, F. Radjai, Y. Monerie, Tensile strength and fracture of porous cemented granular aggregates, European Physical Journal E 35 (2012) 117.

[41] P. Perré, G. Almeida, M. Ayouz, X. Frank, New modelling approaches to predict wood properties from its cellular structure: image-based representation and meshless methods, Annals of Forest Science 73 (2016) $147-162$.

[42] B. Cuq, E. Rondet, J. Abecassis, Food powders engineering, between knowhow and science: Constraints, stakes and opportunities, Powder Technology 208 (2011) 244-251.

[43] J.-C. Charpentier, The triplet "molecular processes-product-process" 
engineering: the future of chemical engineering?, Chemical Engineering Science 57 (2002) 4667-4690.

[44] A. Troccoli, G. Borrelli, P. De-Vita, C. Fares, N. Di-Fonzo, Durum wheat quality: a multidisciplinary concept, Journal of Cereal Science 32 (2000) 99-113.

[45] P. Coussot, C. Ancey, Rheophysical classification of concentrated suspensions and granular pastes, Physical Review E 59 (1999) 4445-4457.

[46] A.-J. Liu, S.-R. Nagel, Jamming is not just cool anymore, Nature 117 (2001) 3-39.

[47] T. Ruiz, E. Rondet, M. Delalonde, J.-P. Desfours, Hydro-textural and consistency surface states of humid granular media, Powder Technology 208 (2011) 409-416.

[48] E. Rondet, M. Delalonde, T. Ruiz, J.-P. Desfours, Hydrotextural and dimensional approach for characterising wet granular media agglomerated by kneading, Chemical Engineering Research and Design 86 (2008) $560-568$.

[49] J. Mellmann, The transverse motion of solids in rotating cylinders forms of motion and transition behavior, Powder Technology 118 (2001) 251-270.

[50] GDR-MIDI, On dense granular flows, European Physical Journal E 14 (2004) 341-336582. 
[51] N. Gravish, P.-B. Umbanhowar, D.-I. Goldman, Force and flow transition in plowed granular media, Physical Review Letters 105 (2010) $128301-$.

[52] S. Mandato, B. Cuq, T. Ruiz, Experimental study of vertical stress profiles in a confined granular bed under static and dynamic conditions, European Physical Journal E (2012) 35-56.

[53] S. Mandato, T. Ruiz, B. Cuq, What is janssen's length doing in an agglomerator?, Powder Technology 238 (2013) 56-63.

[54] A. Duri, F. Mabille, T. Ruiz, Impact of the heap shape formation on the local vertical force profile of ensiled granular materials, submitted to Advanced Powder Technology (2016).

[55] F. Chevoir, J. Roux, F. Da-Cruz, P.-G. Rognon, J.-G. Koval, Friction law in dense granular flow, Powder Technology 190 (2009) 264-268.

[56] A. Goldszal, J. Bousquet, Wet agglomeration of powders: from physics toward process optimization, Powder Technology 117 (2001) 221-231.

[57] S.-M. Iveson, J.-D. Litster, K. Hapgood, B.-J. Ennis, Nucleation, growth and breakage phenomena in wet granulation processes : a review, Powder Technology 117 (2001) 3-39.

[58] E. Rondet, B. Cuq, D. Cassan, T. Ruiz, Agglomeration of wheat powders by a novel reverse wet agglomeration process, Journal of Food Engineering 173 (2016) 92-105. 
[59] E. Rondet, M. Delalonde, T. Ruiz, J.-P. Desfours, Fractal formation description of agglomeration in low shear mixer, Chemical Engineering Journal 164 (2010) 376-382.

[60] A. Barkouti, E. Rondet, M. Delalonde, T. Ruiz, Impact of physicochemical blinder properties on durum wheat powder agglomeration, Journal of Food Engineering 111 (2012) 234-240.

[61] K.-P. Hapgood, J.-D. Litster, R. Smith, Nucleation regime map for liquid bound granules, AIChE Journal 49 (2003) 350-360.

[62] A. Barkouti, M. Delalonde, E. Rondet, T. Ruiz, Structuration of wheat powder by wet agglomeration: case of size association mechanism, Powder Technology 252 (2014) 8-13.

[63] E. Lutton, A. Tonda, N. Boukhelifa, N. Perrot, Complex systems in food science: Human factor issues, in: J. Van Impe (Ed.), FoodSIM, EUROSIS-ETI, 2016.

[64] K. P. Murphy, Dynamic bayesian networks: representation, inference and learning, Ph.D. thesis, University of California, Berkeley, 2002.

[65] J. Pearl, Probabilistic reasoning in intelligent systems: networks of plausible inference, Morgan Kaufmann, 2014.

[66] C. Baudrit, M. Sicard, P.-H. Wuillemin, N. Perrot, Towards a global modelling of the camembert-type cheese ripening process by coupling heterogeneous knowledge with dynamic bayesian networks, Journal of Food Engineering 98 (2010) 283-293. 
[67] N. Perrot, C. Baudrit, J. M. Brousset, P. Abbal, H. Guillemin, B. Perret, E. Goulet, L. Guerin, G. Barbeau, D. Picque, A decision support system coupling fuzzy logic and probabilistic graphical approaches for the agrifood industry: Prediction of grape berry maturity, PLOS ONE 10 (2015) e0134373.

[68] H. Velly, M. Bouix, S. Passot, C. Penicaud, H. Beinsteiner, S. Ghorbal, P. Lieben, F. Fonseca, Cyclopropanation of unsaturated fatty acids and membrane rigidification improve the freeze-drying resistance of lactococcus lactis subsp. lactis tomsc161, Applied microbiology and biotechnology 99 (2015) 907-918.

[69] J. R. Koza, Genetic programming: on the programming of computers by means of natural selection, volume 1, MIT press, 1992 .

[70] M. Schmidt, H. Lipson, Distilling free-form natural laws from experimental data, Science 324 (2009) 81-85. 


\section{Highlights}

- Modeling approaches help understand properties of bioresources and bioproducts.

- Modeling can account for multi-scale phenomena, heterogeneity and variability.

- Physical modeling gives access to collective properties of soft-matter and granular systems.

- Interactive machine-learning approaches can help model complex food systems. 